\title{
Robust Mixture Regression Based on the Mixture of Slash Distributions
}

\author{
Hadi Saboori $^{1, \star}$, Ghobad Barmalzan $^{1}$, Mahdi Doostparast ${ }^{2}$ \\ ${ }^{1}$ Department of Statistics, University of Zabol, Sistan and Baluchestan, Iran \\ ${ }^{2}$ Department of Statistics, Ferdowsi University of Mashhad, Khorasan Razavi, Iran
}

\section{ARTICLE INFO}

\section{Article History}

Received 17 Jun 2017

Accepted 18 Jun 2019

\section{Keywords}

EM algorithm

Normal mixture regression

Outliers

Slash distribution

Mathematics Subject Classification Primary 62J05; Secondary 62F35.

\section{ABSTRACT}

The traditional estimation of Mixture regression models is based on the normal assumption of component errors and thus is sensitive to outliers and heavy-tailed errors. In this paper, we propose a robust Mixture regression models in which a mixture of slash distributions is assumed for the errors. Using the fact that the slash distribution can be written as a scale mixture of a normal and a latent distribution, we also estimate model parameters an expectation-maximization (EM) algorithm. The results of our simulation show that based on AIC and BIC criterion, the proposed robust regression model mixture on slash distribution dominates the robust regression based the normal and the $t$ distribution. Finally, the proposed model is compared with other procedures, based on a real data set.

(C) 2020 The Authors. Published by Atlantis Press SARL. This is an open access article distributed under the CC BY-NC 4.0 license (http://creativecommons.org/licenses/by-nc/4.0/).

\section{INTRODUCTION}

Mixture models are applied in such diverse areas as biology, biometrics, genetics, medicine and marketing, among others. For a brief list of application of the MRM, see Table 1. There are various features of (finite) mixture distributions that make them useful in statistical modeling. For instance, statistical models on the base of finite mixture distributions can capture various properties of real data sets such as multimodality, skewness, kurtosis and unobserved heterogeneity. For more details, see [1-5].

Mixture regression models (hereafter MRM), have been widely used to investigate the relationship between variables coming from several unknown latent homogeneous groups.

The model setting for mixtures of linear regression models can be stated as follows: Let $Z$ be a latent class variable such that given $Z=j$, the (univariate) response $Y$ depends on the $p$-dimensional predictor vector $\mathbf{x}=\left(x_{1}, \ldots, x_{p}\right)$ as

$$
Y=\mathbf{x}^{\top} \beta_{j}+\epsilon_{j}, \quad j=1, \ldots, m,
$$

where $m$ is the number of groups (also called components in mixture models) in the population, the $\beta_{j}$ are unknown $p$-dimensional vectors of regression coefficients and $\epsilon_{j} \sim N\left(0, \sigma_{j}^{2}\right)$ is independent of $\mathbf{x}$. Suppose $P(Z=j)=\pi_{j}$ and $Z$ is independent of $\mathbf{x}$, then the conditional density of $Y$ given $\mathbf{x}$ can be written as

$$
\psi(y ; \mathbf{x}, \delta)=\sum_{j=1}^{m} \pi_{j} \phi\left(y ; \mathbf{x}^{\top} \beta_{j}, \sigma_{j}^{2}\right) .
$$

The log-likelihood function (LLF) for observations $\left\{\left(\mathbf{x}_{1}, y_{1}\right), \ldots,\left(\mathbf{x}_{n}, y_{n}\right)\right\}$ is

$$
\sum_{i=1}^{n} \log \left[\sum_{j=1}^{m} \pi_{j} \phi\left(y_{i} ; \mathbf{x}_{i}^{\top} \beta_{j}, \sigma_{j}^{2}\right)\right],
$$


Table 1 Various application of the mixture regression model (MRM).

\begin{tabular}{lc}
\hline Area of Application of MRM & References \\
\hline Introduced by & {$[6]$} \\
Marketing & {$[7-9]$} \\
Economics & {$[10,11]$} \\
Agriculture & {$[12]$} \\
Nutrition & {$[13]$} \\
Psychometrics & {$[14]$} \\
\hline
\end{tabular}

where $\phi\left(\cdot ; \mu, \sigma^{2}\right)$ is the density function of the normal distribution with mean $\mu$ and variance $\sigma^{2}$, hereafter denote by $N\left(\mu, \sigma^{2}\right)$ and $\delta=$ $\left(\delta_{1}^{\top}, \ldots, \delta_{m}^{\top}\right)^{\top}$ with $\delta_{j}=\left(\pi_{j}, \beta_{j}, \sigma_{j}^{2}\right)^{\top}$.

The computation can be further simplified based on the fact that the slash distribution can be considered as a scale mixture of normal distributions. Let $U$ be the latent variable such that

$$
\epsilon \mid u \sim N\left(0, \sigma^{2} / u^{2}\right) \text { and } U \sim \operatorname{Beta}(q, 1),
$$

where corresponding density function to $\operatorname{Beta}(\alpha, \beta)$ is

$$
h(u ; \alpha, \beta)=b(\alpha, \beta) u^{\alpha-1}(1-u)^{\beta-1}, \quad 0<u<1,
$$

and $b(\alpha, \beta)$ is the Beta function. Then, marginally $\epsilon$ has a slash distribution with parameter $\mathrm{q}$ and scale parameter $\sigma$.

The unknown parameters in the model (2) can be estimated maximizing the LLF (3). Since the LLF (3) complicated, the maximum likelihood estimations (MLEs) are derived by maximizing (3) numerically. Among numerically methods for maximizing (3), the EM algorithm is commonly used; see [15].

The MLE $\hat{\delta}$ in (3) works well when the error distribution is normal. However, the normality based MLE is sensitive to outliers or heavytailed error distributions. To overcome this problem [16] and [17] proposed a weight factor for each data point to robustify the estimation procedure for MRM. Neykov et al. [18] proposed robust fitting of mixtures using the trimmed likelihood estimator (TLE). Bai et al. [19] proposed a modified EM algorithm to robustly estimate the mixture regression parameters by replacing the least squares criterion in M-step with a robust criterion. Through a simulation study, they show that their proposed estimate is robust when the data have outliers or the error distribution has heavy tails. Bashir and Carter [20] extended the idea of the S-estimator to mix linear regression models.

Yao et al. [21] proposed a robust estimation procedure for mixture linear regression models by assuming that the error terms follow the $t$ Student distribution. Using the skew-normal distribution defined by [22,23] proposed a version of the mixture linear regression model. Dogru and Arslan [24] also proposed a new version of MRM modeling using the least trimmed squares (LTSs) estimation method.

Recently, researchers proposed the MRM based on skew and/or heavy tailed distributions. Lin et al. [25,4] respectively, using skew $\mathrm{t}$ and multivariate skew t-Normal distributions for this regression model. Song et al. [26] use the Laplace distribution. Zeller et al. [27] proposed a robust MRM based on the scale mixtures of skew-normal distributions. These authors also developed a simple EM-type algorithm to perform maximum likelihood inference of the parameters of the proposed model. In order to examine the robust aspect of this flexible model against outlying observations, some simulation studies have also been proposed. For example [28] proposed MRM using the mixture of different distributions. Dogru and Arslan [29,30] used other skew distribution for this regression model.

In recent years, attempts have been made by many researchers to propose flexible statistical models for fitting various data sets. Among the well-known distribution functions, the slash distribution, introduced by [31], provides more flexibility. See Section 2.

In this paper, we propose a robust MRM based on the slash distribution by assuming the mixture of slash distributions errors. Similar to the traditional M-estimate for linear regression (see [32]), the proposed estimate is expected to be sensitive to high leverage outliers. To overcome this problem, we also propose a modified version of the new method by fitting the new model to the data after adaptively trimming high leverage points. Compared to the TLE, the proportion of trimming of the new proposed model is data adaptive instead of a fixed value. Using a simulation study and real data application, we compare the new method to some existing methods, and demonstrate the effectiveness of the proposed method.

Therefore, the rest of this paper is organized as follows: In Section 2 the slash distribution and its properties are reviewed. In Section 3, we introduce our new robust mixture linear regression models based on the slash distribution and then develop the respective EM algorithm. In Section 4, we propose to further improve the robustness of the proposed method against high leverage outliers by adaptively trimming high leverage points. Also in this section, with simulation study, we compare the proposed method to the traditional MLE and some other robust methods by using a simulation study. Finally in Section 5, these results have been applied in real data.

The slash distribution with parameter $q$ is defined as the ratio of two independent random variables $Z$ and $U^{1 / q}$, where $Z$ is the standard normal random variable and $U$ has uniform distribution in $(0,1)$. The main feature of the slash distribution is to have greater flexibility in 
the degree of kurtosis with respect to the normal distributions. Other advantages of this distribution are the properties of symmetry, heavy tails and the inclusion of the normal family as a limit case when $q$ tends to infinity. Also as this density is less peaked, in the center, than normal density, it is more realistic in representing data. For various properties of the slash distribution and its applications, we refer the interested reader to [33,34] and [35]. Also, Alcantara and Cysneiros [36] and [36] proposed a linear regression model with slash errors.

\section{THE SLASH DISTRIBUTION}

In this section, in order to more robustly estimate the mixture regression parameters, we assume that the error density function in (1) is a slash distribution with parameter $q_{j}>0$ and scale parameter $\sigma_{j}>0$, as following:

$$
f\left(\epsilon_{j} ; \sigma_{j}, q_{j}\right)=\frac{q_{j}}{\sigma_{j}} \int_{0}^{1} u^{q_{j}} \phi\left(\frac{u \epsilon_{j}}{\sigma_{j}}\right) d u, \quad \epsilon_{j} \in \mathbb{R}, \quad j=1, \ldots, m .
$$

The MRM with slash distribution can be formulated in a similar way to the model defined in (2) as follows:

$$
g(y ; \mathbf{x}, \Theta)=\sum_{j=1}^{m} \pi_{j} f\left(y-\mathbf{x}^{\top} \beta_{j} ; \sigma_{j}, q_{j}\right)
$$

where $f(\cdot ; \sigma, q)$ is the density function of the slash distribution given by (4) and $\Theta=\left(\theta_{1}^{\top}, \ldots, \theta_{m}^{\top}\right)^{\top}$ with $\theta_{j}=\left(\beta_{j}, \sigma_{j}, \pi_{j}\right)^{\top}$. The unknown parameter vector $\Theta$ in (5), given observations $\left(\mathbf{x}_{1}, y_{1}\right), \ldots,\left(\mathbf{x}_{n}, y_{n}\right)$, is

$$
\hat{\Theta}=\arg \max _{\Theta} \sum_{i=1}^{n} \log \left\{\sum_{j=1}^{m} \pi_{j} f\left(y_{i}-\mathbf{x}_{i}^{\top} \beta_{j} ; \sigma_{j}, q_{j}\right)\right\} .
$$

Note that the LLF (6) is unbounded if one of the observations $y_{i}$ is equal to $\mathbf{x}_{i}^{\top} \beta_{j}$ and $\sigma_{j} \rightarrow 0$. For more details, see [37,38] and [39]. Also notice that, the maximizer of (6) does not have an explicit solution, so we propose to use an EM-type algorithm (see [15]). For a gentle tutorial on the EM algorithm and its applications to parameter estimation for mixture models, see [40] and [41].

\section{MAXIMUM LIKELIHOOD ESTIMATION}

In this subsection, we present an EM algorithm for the ML estimation of the MRM with the slash distribution given by $(5)$. For $j=1, \ldots, m$, and $i=1, \ldots, n$, denote $z_{i j}$ as latent Bernoulli variables such that

$$
z_{i j}= \begin{cases}1, & \text { if the } i^{t h} \text { observation is from the } j^{\text {th }} \text { component, } \\ 0, & \text { otherwise. }\end{cases}
$$

If the complete data set $\mathbf{T}=\left\{\left(\mathbf{x}_{\mathbf{i}}, y_{i}, z_{i j}\right) ; i=1, \ldots, n, j=1, \ldots, m\right\}$ is observable, the complete LLF of $\Theta$ can be written as

$$
\ell_{c}(\Theta ; \mathbf{T})=\sum_{i=1}^{n} \sum_{j=1}^{m} z_{i j} \log \left\{\pi_{j} f\left(y_{i}-\mathbf{x}_{i}^{\top} \beta_{j} ; \sigma_{j}, q_{j}\right)\right\} .
$$

Based on the theory of EM algorithm, in E-step, given current estimate $\Theta^{(k)}$ at the $k^{\text {th }}$ step, we calculate $E\left(\ell_{c}(\Theta ; \mathbf{T}) \mid \mathbf{X}, \mathbf{y}, \Theta^{(k)}\right)$ which simplifies to the calculation of $E\left(Z_{i j} \mid \mathbf{X}, \mathbf{y}, \Theta^{(k)}\right)$. Then in M-step, we find the maximizer of

$$
E\left(\ell_{c}(\Theta ; \mathbf{T}) \mid \mathbf{X}, \mathbf{y}, \Theta^{(k)}\right)=\sum_{i=1}^{n} \sum_{j=1}^{m} E\left(Z_{i j} \mid \mathbf{X}, \mathbf{y}, \Theta^{(k)}\right) \log \left\{\pi_{j} f\left(y_{i}-\mathbf{x}_{i}^{\top} \beta_{j} ; \sigma_{j}, q_{j}\right)\right\} .
$$

Note that the above maximizer does not have explicit solutions for $\beta_{j}, \sigma_{j}$ and $q_{j}$.

Since, we can simplify the computation of M-step of the proposed EM algorithm by introducing another latent variable $U$, therefore the complete likelihood for $(\mathbf{y}, \mathbf{u}, \mathbf{z})$ given $\mathbf{X}$ is

$$
\begin{aligned}
\ell_{c}^{*}(\Theta ; \mathbf{X}, \mathbf{y}, \mathbf{z}, \mathbf{u})= & \sum_{i=1}^{n} \sum_{j=1}^{m} z_{i j} \log \left\{\pi_{j} \phi\left(y_{i}-\mathbf{x}_{i}^{\top} \beta_{j} ; 0, \sigma_{j}^{2} / u_{i j}^{2}\right) h\left(u_{i j} ; q_{j}, 1\right)\right\} \\
= & \sum_{i=1}^{n} \sum_{j=1}^{m} z_{i j} \log \pi_{j}+\sum_{i=1}^{n} \sum_{j=1}^{m} z_{i j} \log q_{j}+\sum_{i=1}^{n} \sum_{j=1}^{m} z_{i j}\left(q_{j}-1\right) \log u_{i j} \\
& +\sum_{i=1}^{n} \sum_{j=1}^{m} z_{i j}\left\{-\frac{1}{2} \log \left(2 \pi \sigma_{j}^{2}\right)+\log u_{i j}-\frac{u_{i j}^{2}}{2 \sigma_{j}^{2}}\left(y_{i}-\mathbf{x}_{i}^{\top} \beta_{j}\right)^{2}\right\},
\end{aligned}
$$


where $\mathbf{u}=\left(u_{11}, \ldots, u_{n m}\right)$ is independent of $\mathbf{z}=\left(z_{11}, \ldots, z_{n m}\right)$.

In the E-step on the $(k+1)^{\text {th }}$ iteration, we need to calculate the conditional expectation of the LLF of complete data, which is $E\left(\ell_{c}^{*}(\Theta ; \mathbf{X}, \mathbf{y}, \mathbf{z}, \mathbf{u}) \mid \mathbf{X}, \mathbf{y}, \Theta^{(k)}\right)$. Based on the above argument, the E-step requires the calculations of $E\left(Z_{i j} \mid \mathbf{X}, \mathbf{y}, \Theta^{(k)}\right)$, $E\left(U_{i j}^{2} \mid \mathbf{X}, \mathbf{y}, z_{i j}=1, \Theta^{(k)}\right)$ and $E\left(\log U_{i j} \mid \mathbf{X}, \mathbf{y}, z_{i j}=1, \Theta^{(k)}\right)$. Thus, the EM algorithm can be written as

1. Choose some initial value $\Theta^{(0)}=\left(\beta_{1}^{(0)}, q_{1}^{(0)}, \sigma_{1}^{(0)}, \pi_{1}^{(0)}, \ldots, \beta_{m}^{(0)}, q_{m}^{(0)}, \sigma_{m}^{(0)}, \pi_{m}^{(0)}\right)^{\top}$.

2. E-step: On the $(k+1)^{\text {th }}$ iteration, according to Bayes theorem, we can compute conditional expectations as follows:

$$
\begin{gathered}
p_{i j}^{(k+1)}=E\left(Z_{i j} \mid \mathbf{X}, \mathbf{y}, \Theta^{(k)}\right)=\frac{\pi_{j}^{(k)} f\left(y_{i}-\mathbf{x}_{i}^{\top} \beta_{j}^{(k)} ; \sigma_{j}^{(k)}, q_{j}^{(k)}\right)}{\sum_{l=1}^{m} \pi_{l}^{(k)} f\left(y_{i}-\mathbf{x}_{i}^{\top} \beta_{l}^{(k)} ; \sigma_{l}^{(k)}, q_{l}^{(k)}\right)}, \\
l_{i j}^{(k+1)}=E\left(\log U_{i j} \mid \mathbf{X}, \mathbf{y}, z_{i j}=1, \Theta^{(k)}\right) \\
=\frac{\int_{0}^{1} \log (u) q_{j}^{(k)} u^{q_{j}^{(k)}} \exp \left\{-\frac{u^{2}\left(y_{i}-\mathbf{x}_{i}^{\top} \beta_{j}^{(k)}\right)^{2}}{2\left(\sigma_{j}^{(k)}\right)^{2}}\right\}}{\sigma_{j}^{(k)} \sqrt{2 \pi} f\left(y_{i}-\mathbf{x}_{i}^{\top} \beta_{j}^{(k)} ; \sigma_{j}^{(k)}, q_{j}^{(k)}\right)} d u,
\end{gathered}
$$

and

$$
\begin{aligned}
e_{i j}^{(k+1)}= & E\left(U_{i j}^{2} \mid \mathbf{X}, \mathbf{y}, z_{i j}=1, \Theta^{(k)}\right) \\
& = \begin{cases}\frac{q_{j}^{(k)} 2^{\frac{q_{j}^{(k)}}{2}}\left(\frac{\left(y_{i}-\mathbf{x}_{i}^{\top} \beta_{j}^{(k)}\right)^{2}}{\left(\sigma_{j}^{(k)}\right)^{2}}\right)^{-\frac{q_{j}^{(k)}+3}{2}} \Gamma\left(\frac{q_{j}^{(k)}+3}{2}\right)}{\sigma_{j}^{(k)} \sqrt{\pi} f\left(y_{i}-\mathbf{x}_{i}^{\top} \beta_{j}^{(k)} ; \sigma_{j}^{(k)}, q_{j}^{(k)}\right)\left[G\left(\frac{\left(y_{i}-\mathbf{x}_{i}^{\top} \beta_{j}^{(k)}\right)^{2}}{2\left(\sigma_{j}^{(k)}\right)^{2}} ; \frac{q_{j}^{(k)}+3}{2}, 1\right)\right]^{-1},} & y_{i}-\mathbf{x}_{i}^{\top} \beta_{j}^{(k)} \neq 0, \\
\frac{q_{j}^{(k)}}{q_{j}^{(k)}+3} \frac{1}{\sigma_{j}^{(k)} \sqrt{2 \pi} f\left(0 ; \sigma_{j}^{(k)}, q_{j}^{(k)}\right)}, & y_{i}-\mathbf{x}_{i}^{\top} \beta_{j}^{(k)}=0,\end{cases}
\end{aligned}
$$

where $\Gamma(\cdot)$ and $G(\cdot ; r, 1)$ are the complete gamma function and the cumulative distribution function of the gamma distribution with parameters shape $r$ and scale 1 , respectively.

3. M-step: On the $(k+1)^{\text {th }}$ iteration, compute the estimator of parameters which maximize the expected complete log-likelihood. The estimators can be written as

$$
\begin{gathered}
\pi_{j}^{(k+1)}=\frac{1}{n} \sum_{i=1}^{n} p_{i j}^{(k+1)}, \\
\beta_{j}^{(k+1)}=\left(\sum_{i=1}^{n} \mathbf{x}_{i} \mathbf{x}_{i}^{\top} p_{i j}^{(k+1)} e_{i j}^{(k+1)}\right)^{-1}\left(\sum_{i=1}^{n} \mathbf{x}_{i} y_{i} p_{i j}^{(k+1)} e_{i j}^{(k+1)}\right), \\
\sigma_{j}^{(k+1)}=\left\{\frac{\sum_{i=1}^{n} p_{i j}^{(k+1)} e_{i j}^{(k+1)}\left(y_{i}-\mathbf{x}_{i}^{\top} \beta_{j}^{(k+1)}\right)^{2}}{\sum_{i=1}^{n} p_{i j}^{(k+1)}}\right\}^{1 / 2},
\end{gathered}
$$

and

$$
q_{j}^{(k+1)}=-\frac{\sum_{i=1}^{n} p_{i j}^{(k+1)}}{\sum_{i=1}^{n} p_{i j}^{(k+1)} l_{i j}^{(k+1)}} .
$$


4. Repeat the E-step and the M-step until the convergence is obtained. One stopping rule that we can choose is to stop the iteration when the change of the likelihood value is smaller than $10^{-} 6$ or runs are more than 500 .

Remarke: If we further assume that all $\sigma_{j}$ 's are equal, then in the M-step, we can update $\sigma$ by

$$
\sigma^{(k+1)}=\left\{\frac{\sum_{i=1}^{n} \sum_{j=1}^{m} p_{i j}^{(k+1)} e_{i j}^{(k+1)}\left(y_{i}-\mathbf{x}_{i}^{\top} \beta_{j}^{(k+1)}\right)^{2}}{n}\right\}^{1 / 2} .
$$

\subsection{Initial Point in the EM Algorithm}

It is well known that mixture models may provide a multimodal LLF. In this sense, the method of MLE through EM algorithm may not give maximum global solutions if the starting values are far from the real parameter values. Thus, the choice of starting values for the EM algorithm in the mixture context plays an important role in parameters estimation. In our example and simulation studies, we consider the following procedure for the slash MRM.

- Partition the sample into $m$ groups using the K-means clustering algorithm (see [42]).

- Compute the proportion of data points belonging to the same cluster $j$, say $p_{j}^{(0)}, j=1, \ldots, m$. This is the initial value for $p_{j}$.

- For each group $j$, compute the initial values $\beta_{j}^{(0)}$ using the method of least square. Then, use the residuals of each group to compute initial values $\sigma_{j}^{(0)}$ and $q_{j}^{(0)}$.

\subsection{Evaluation of the Standard Errors from the EM-Algorithm}

In this subsection, we use the results of [43] to obtain the standard errors of the estimators from the EM-algorithm. For evaluation of the standard errors, one can also see [44]. Let $\mathbf{v}=(\mathbf{X}, \mathbf{y}, \mathbf{z}, \mathbf{u})$, then the elements of information matrix $\mathbf{I}_{j}^{*}\left(\theta_{j} ; \mathbf{v}\right)=-\left[\partial^{2} \ell_{c}^{*}(\Theta ; \mathbf{v}) / \partial \theta_{j}^{2}\right]$, for $j=1, \ldots, m$, are given in Appendix A.

Taking the conditional expectation of $\mathbf{I}_{j}^{*}\left(\theta_{j} ; \mathbf{v}\right)$ given $(\mathbf{X}, \mathbf{y})$, we obtain the $(p+3) \times(p+3)$ matrix

$$
\ell_{c}^{j}\left(\theta_{j} ; \mathbf{X}, \mathbf{y}\right)=E\left(\mathbf{I}_{j}^{*}\left(\theta_{j} ; \mathrm{v}\right) \mid \mathbf{X}, \mathbf{y}\right)=\left[c_{k l}^{j}\right]
$$

that the elements of matrix $\left[c_{k l}^{j}\right]$ are presented in Appendix B.

Moving now to the computation of $\left.\ell_{m}^{j}\left(\hat{\theta}_{j} ; \mathbf{X}, \mathbf{y}\right)\right)$ as

$$
\ell_{m}^{j}\left(\hat{\theta}_{j} ; \mathbf{X}, \mathbf{y}\right)=\operatorname{Var}\left(U_{c}^{j}\left(\mathbf{v} ; \hat{\theta}_{j}\right) \mid \mathbf{X}, \mathbf{y}\right)=\left[v_{k l}^{j}\right]
$$

where $U_{c}^{j}\left(\mathbf{v} ; \hat{\theta}_{j}\right)=\left(\frac{\partial \ell_{c}^{*}(\Theta ; \mathbf{v})}{\partial \beta_{j}}, \frac{\partial \ell_{c}^{*}(\Theta ; \mathbf{v})}{\partial q_{j}}, \frac{\partial e_{c}^{*}(\Theta ; \mathbf{v})}{\partial \sigma_{j}}, \frac{\partial e_{c}^{*}(\Theta ; \mathbf{v})}{\partial \pi_{j}}\right)^{\top}$ and the elements of matrix $\left[v_{k l}^{j}\right]$ are given by Appendix C. Note that

$$
E\left(g\left(U_{i}\right) \mid \mathbf{y}, \mathbf{X}, z_{i j}=1\right)=\int_{0}^{1} g\left(u_{i}\right) \frac{q_{j} u_{i}^{q_{j}} \exp \left\{-\frac{u_{i}^{2}\left(y_{i}-\mathbf{x}_{i}^{T} \beta_{j}\right)^{2}}{2 \sigma_{j}^{2}}\right\}}{\sigma_{j} \sqrt{2 \pi} f\left(y_{i}-\mathbf{x}_{i}^{T} \beta_{j} ; \sigma_{j}, q_{j}\right)} d u_{i}
$$

where the $g(\cdot)$ is real function of $u_{i}$ from (15) and (16), we obtain the observed information matrix $\hat{I}=\left[\left[I_{j}\left(\hat{\theta}_{j} ; \mathbf{X}, \mathbf{y}\right)\right]\right]$

$$
\mathrm{I}_{j}\left(\hat{\theta}_{j} ; \mathbf{X}, \mathbf{y}\right)=\ell_{c}^{j}\left(\hat{\theta}_{j} ; \mathbf{X}, \mathbf{y}\right)-\ell_{m}^{j}\left(\hat{\theta}_{j} ; \mathbf{X}, \mathbf{y}\right)
$$

The standard errors of the MLEs of the EM-algorithm are the inverse of square root of the diagonal elements of the $\mathrm{I}_{j}\left(\hat{\theta}_{j} ; \mathbf{X}, \mathbf{y}\right)$. 


\section{SIMULATION STUDY}

To assess the finite sample performance of the proposed model and the estimation procedure, we conducted an simulation study. In the EM algorithm, it is well khnown that label switching is always an issue when evaluating different estimation methods in mixture models, and there are no widely accepted labeling standards. In this simulation study, we choose the labels by minimizing the distance to the true parameter values. However, more research on choosing labels is needed (see [45]). Also, we considered equal variance for all components. The reason is that, if the variances are not same, the LLF (7) is unbounded and tends to infinity if one observation lies exactly on one component line and the corresponding variance vanishes, which makes the simulation very unstable.

\subsection{Evaluate Robustness of the MRM}

We use the method that proposed by [21] for evaluating robustness of the MRM. They proposed a trimmed version of the new method by fitting the new model to the data after adaptively trimming high leverage points. In addition, unlike TLE (see [18]), the proportion of trimming of the new method is data adaptive instead of a fixed value (For more explain, see [21]). Let $\mathbf{X}=\left(\mathbf{x}_{1}, \ldots, \mathbf{x}_{n}\right)^{\top}$ and $h_{i i}$ be the $i^{\text {th }}$ diagonal element of $\mathbf{H}$, where $\mathbf{H}=\mathbf{X}\left(\mathbf{X}^{\top} \mathbf{X}\right)^{-1} \mathbf{X}^{\top}$. The element $h_{i i}$ is called the leverage for the $i^{\text {th }}$ predictor $\mathbf{x}_{i}$ and $\mathbf{x}_{i}$ is considered a high leverage point if $h_{i i}$ is large. Note however

$$
h_{i i}=n^{-1}+(n-1)^{-1} M D_{i},
$$

where

$$
M D_{i}=\left(\mathbf{x}_{i}-\overline{\mathbf{x}}\right)^{\top} \mathbf{S}^{-1}\left(\mathbf{x}_{i}-\overline{\mathbf{x}}\right),
$$

is the Mahalanobis distance, $\overline{\mathbf{x}}$ is the sample mean of $\mathbf{x}_{i}{ }^{\prime}$, and $\mathbf{S}$ is the sample covariance of $\mathbf{x}_{i}{ }^{\prime}$ ' (without the intercept 1 ). It is well known that $\overline{\mathbf{x}}$ and $\mathbf{S}$ are not resistant to outliers and might create the masking effect (see [46]), i.e., some high leverage points might not be identified due to the influence of other high-leverage points. In order to overcome this, a modified Mahalanobis distance is proposed

$$
M D_{i}=\left(\mathbf{x}_{i}-\mathbf{m}(\mathbf{X})\right)^{\top} \mathbf{C}(\mathbf{X})^{-1}\left(\mathbf{x}_{i}-\mathbf{m}(\mathbf{X})\right),
$$

where $\mathbf{m}(\mathbf{X})$ and $\mathbf{C}(\mathbf{X})$ are robust estimates of location and scatter for $\mathbf{X}$ (after removing the first column 1s).

Suppose that $\left\{\left(x_{1 i}, x_{2 i}, y_{i}\right), i=1, \ldots, n\right\}$ is a random sampled from the following model

$$
Y= \begin{cases}0+X_{1}+X_{2}+\epsilon_{1}, & \text { if } Z=1 \\ 0-X_{1}-X_{2}+\epsilon_{2}, & \text { if } Z=2,\end{cases}
$$

where $Z$ is a component indicator of $Y$ with $P(Z=1)=1-P(Z=2)=0.25, X_{1} \sim N(0,1), X_{2} \sim N(0,1)$, and $\epsilon_{1}$ and $\epsilon_{2}$ have the same distribution as $\epsilon$. To see the effects of different distributions of $\epsilon$ and high leverage outliers in $\mathbf{x}$-direction on various estimation methods, we considered the following six cases:

$$
\begin{array}{ll}
\text { Case (I): } & \epsilon \sim N(0,1), \text { standard normal distribution. } \\
\text { Case (II): } & \epsilon \sim \operatorname{slash}(1,2) \text {, slash distribution with parameter } q=2 \text { and scale parameter } \sigma=1 . \\
\text { Case (III): } & \epsilon \sim t_{1}, t \text {-distribution with } 1 \text { degree of freedom. } \\
\text { Case (IV): } & \epsilon \sim l(0,1), \text { Laplace distribution with mean } 0 \text { and variance } 1 . \\
\text { Case (V): } & \epsilon \sim 0.95 N(0,1)+0.05 N(0,25) \text {, a mixture of two normal distributions. } \\
\text { Case (VI): } & \epsilon \sim N(0,1) \text { with } 5 \% \text { high leverage outliers being } X_{1}=X_{2}=20 \text { and } Y=100 .
\end{array}
$$

Case (I) is often used to evaluate the efficiency of different estimation methods compared to the traditional MLE when the error is normally distributed and there is no outliers. For Case (II), the estimation methods proposed in this paper will provide the MLE of unknown parameters, which, as in the first case, would serve as a baseline to evaluate the performance of other estimation procedures. Cases (III) and (IV) are heavy tailed distribution and are often used in the literature to mimic the outlier situations. Case (V) would produce $5 \%$ data likely to be low leverage outliers, and in Case (VI), 5\% of the observations are replicated serving as the high leverage outliers, which will be used to check the robustness of estimation procedures against the high leverage outliers.

Five estimation methods were compared in the simulation study: (1) the maximum likelihood method based on the normality assumption (MLE); (2) the TLE proposed by [18]; (3) the robust modified EM algorithm based on bisquare (Bisquare) proposed by [19]; (4) the proposed robust EM mixture regression method based on the slash distribution (Mixregs); (5) the trimmed mixture regression method based on the slash distribution (Mixregs-MCD), with MCD trimming method. In the iterative numerical methods, the iteration is terminated when the change in the likelihood function is less than $10^{6}$. 
Tables 2-5 report the mean squared errors (MSE) and the absolute bias (Bias) of the parameter estimates for each estimation method for sample sizes $n=50,100,200$ and $n=400$, respectively. The number of replicates is 5000. As shown in Tables 2-5, in Case (I) through (IV), Mixregs and Mixregs-MCD performed at a level that is better or equal to the other three methods. In Case $(\mathrm{V})$ where there are high leverage outliers, Mixregs-MCD also outperformed the other four methods.

Table 2 Bias and MSE (in parentheses) of point estimates for $n=50$.

\begin{tabular}{|c|c|c|c|c|c|c|c|}
\hline True & MLE & TLE & Bisquare & Mixregt & Mixregt-MCD & Mixregs & Mixregs-MCD \\
\hline \multicolumn{8}{|c|}{ Case (I): $\varepsilon \sim N(0,1)$} \\
\hline$\beta_{10}: 0$ & $0.0035(0.4785)$ & $0.0109(0.6015)$ & $0.0040(0.4875)$ & $0.0032(0.4561)$ & $0.0071(0.5167)$ & $0.0001(0.3494)$ & $0.0056(0.3770)$ \\
\hline$\beta_{11}: 1$ & $0.1528(0.5638)$ & $0.8904(1.5952)$ & $0.4678(0.9193)$ & $0.2377(0.6354)$ & $0.2862(0.7588)$ & $0.0495(0.3492)$ & $0.0722(0.4207)$ \\
\hline$\beta_{12}: 1$ & $0.1366(0.5285)$ & $0.8904(1.5965)$ & $0.4586(0.8733)$ & $0.2217(0.6059)$ & $0.2696(0.7172)$ & $0.0393(0.3625)$ & $0.0612(0.4490)$ \\
\hline$\beta_{20}: 0$ & $0.0006(0.0869)$ & $0.0053(0.2304)$ & $0.0038(0.1296)$ & $0.0019(0.0981)$ & $0.0059(0.1269)$ & $0.0012(0.0455)$ & $0.0024(0.0544)$ \\
\hline$\beta_{21}^{20}:-1$ & $0.0058(0.0627)$ & $0.0212(0.1404)$ & $0.0277(0.0879)$ & $0.0084(0.0702)$ & $0.0068(0.0985)$ & $0.0074(0.0472)$ & $0.0027(0.0661)$ \\
\hline$\beta_{22}^{21}:-1$ & $0.0160(0.0680)$ & $0.0275(0.1278)$ & $0.0432(0.0906)$ & $0.0212(0.0663)$ & $0.0246(0.0933)$ & $0.0013(0.0483)$ & $0.0007(0.0654)$ \\
\hline$\pi_{1}: 0.25$ & $0.0326(0.0120)$ & $0.1413(0.0374)$ & $0.0891(0.0259)$ & $0.0510(0.0149)$ & $0.0626(0.0182)$ & $0.0003(0.0130)$ & $0.0068(0.0151)$ \\
\hline \multicolumn{8}{|c|}{ Case (II): $\varepsilon \sim \operatorname{slash}(0,1)$} \\
\hline$\beta_{10}: 0$ & $0.0592(52.9240)$ & $0.0066(1.5574)$ & $0.0065(1.5919)$ & $0.0178(3.9062)$ & $0.0531(4.1787)$ & $0.0160(0.7033)$ & $0.0200(0.8057)$ \\
\hline$\beta_{11}: 1$ & $0.5294(65.3548)$ & $0.7148(1.9506)$ & $0.5117(1.6059)$ & $0.3411(4.5422)$ & $0.3415(4.9709)$ & $0.0460(0.6372)$ & $0.0646(0.8130)$ \\
\hline$\beta_{12}: 1$ & $0.2843(39.1912)$ & $0.7195(1.8359)$ & $0.5054(1.6077)$ & $0.4040(4.0941)$ & $0.3734(4.7012)$ & $0.0409(0.6399)$ & $0.0537(0.8949)$ \\
\hline$\beta_{20}: 0$ & $0.0018(38.2963)$ & $0.0035(0.8519)$ & $0.0112(0.7225)$ & $0.0548(2.7391)$ & $0.0200(2.6460)$ & $0.0020(0.0799)$ & $0.0028(0.0895)$ \\
\hline$\beta_{21}^{20}:-1$ & $1.0935(36.3988)$ & $0.0835(0.6123)$ & $0.1200(0.6647)$ & $0.2309(3.0690)$ & $0.2967(4.3152)$ & $0.0073(0.0898)$ & $0.0082(0.1115)$ \\
\hline$\beta_{22}:-1$ & $1.2521(41.2024)$ & $0.0899(0.5964)$ & $0.1553(0.5842)$ & $0.2122(2.8183)$ & $0.2575(3.6373)$ & $0.0023(0.0891)$ & $0.0042(0.1058)$ \\
\hline$\pi_{1}: 0.25$ & $0.1820(0.1555)$ & $0.1717(0.0547)$ & $0.1528(0.0570)$ & $0.1505(0.0643)$ & $0.1592(0.0695)$ & $0.0122(0.0278)$ & $0.0122(0.0288)$ \\
\hline \multicolumn{8}{|c|}{ Case (III): $\varepsilon \sim t_{1}$} \\
\hline$\beta_{10}: 0$ & $11.6090(501705.6719)$ & $0.1484(36.6703)$ & $0.0082(2.7508)$ & $0.0009(9.2711)$ & $0.0010(10.8682)$ & $0.0161(1.6247)$ & 0.0444(1.7095) \\
\hline$\beta_{11}^{10}: 1$ & $21.7989(49070.7569)$ & $0.1158(37.1632)$ & $0.5407(3.0793)$ & $0.4597(12.4083)$ & $0.2960(22.9286)$ & $0.0536(1.6220)$ & $0.0279(2.0650)$ \\
\hline$\beta_{12}: 1$ & $44.2708(1982026.0620)$ & $0.0477(19.2282)$ & $0.5466(2.7890)$ & $0.3747(17.2967)$ & $0.3317(20.0879)$ & $0.0122(1.9936)$ & $0.0154(2.3890)$ \\
\hline$\beta_{20}: 0$ & $6.5844(1410067.1062)$ & $0.0457(13.4585)$ & $0.0426(8.4725)$ & $0.0329(13.4541)$ & $0.0239(14.6770)$ & $0.0048(0.1757)$ & $0.0021(0.1658)$ \\
\hline$\beta_{21}^{20}:-1$ & $48.3717(2540865.2077)$ & $0.5829(15.6338)$ & $0.1778(2.0443)$ & $0.4748(13.1418)$ & $0.5896(13.8616)$ & $0.0002(0.1785)$ & $0.0071(0.2151)$ \\
\hline$\beta_{22}:-1$ & $46.2134(713106.5159)$ & $0.6152(14.6803)$ & $0.2375(22.8824)$ & $0.4430(13.5257)$ & $0.4694(18.4484)$ & $0.0063(0.1780)$ & $0.0109(0.2317)$ \\
\hline$\pi_{1}: 0.25$ & $0.2414(0.2483)$ & $0.1746(0.1025)$ & $0.1844(0.0760)$ & $0.1769(0.0677)$ & $0.1829(0.0724)$ & $0.0193(0.0436)$ & $0.0226(0.0443)$ \\
\hline \multicolumn{8}{|c|}{ Case (IV): $\varepsilon \sim l(0,1)$} \\
\hline$\beta_{10}: 0$ & $0.0097(0.7824)$ & $0.0100(0.5476)$ & $0.0222(0.4867)$ & $0.0052(0.4622)$ & $0.0059(0.5169)$ & $0.0061(0.2894)$ & $0.0090(0.3205)$ \\
\hline$\beta_{11}: 1$ & $0.1221(0.8182)$ & $0.6228(1.1672)$ & $0.4430(0.9152)$ & $0.1946(0.6037)$ & $0.2138(0.6899)$ & $0.0309(0.2877)$ & $0.0716(0.4048)$ \\
\hline$\beta_{12}: 1$ & $0.1172(0.7817)$ & $0.6214(1.1486)$ & $0.4314(0.8522)$ & $0.1882(0.5904)$ & $0.2190(0.7188)$ & $0.0450(0.2821)$ & $0.0514(0.4334)$ \\
\hline$\beta_{20}^{12}: 0$ & $0.0009(0.1970)$ & $0.0033(0.1314)$ & $0.0020(0.1105)$ & $0.0018(0.0652)$ & $0.0013(0.0824)$ & $0.0009(0.0217)$ & $0.0005(0.0353)$ \\
\hline$\beta_{21}^{20}:-1$ & $0.0229(0.1755)$ & $0.0182(0.1083)$ & $0.0241(0.0728)$ & $0.0093(0.0687)$ & $0.0149(0.0932)$ & $0.0012(0.0243)$ & $0.0036(0.0426)$ \\
\hline$\beta_{22}^{21}:-1$ & $0.0413(0.1677)$ & $0.0269(0.0955)$ & $0.0457(0.0788)$ & $0.0207(0.0669)$ & $0.0242(0.0956)$ & $0.0018(0.0246)$ & $0.0070(0.0452)$ \\
\hline$\pi_{1}: 0.25$ & $0.0355(0.0181)$ & $0.0953(0.0269)$ & $0.0872(0.0249)$ & $0.0450(0.0129)$ & $0.0530(0.0156)$ & $0.0086(0.0138)$ & $0.0112(0.0147)$ \\
\hline \multicolumn{8}{|c|}{ Case $(\mathrm{V}): \varepsilon \sim 0.95 N(0,1)+0.05 N(0,25)$} \\
\hline$\beta_{10}: 0$ & $0.0239(5.1865)$ & $0.0070(0.6854)$ & $0.0129(0.6047)$ & $0.0077(1.1336)$ & $0.0053(1.2544)$ & $0.0000(0.2890)$ & $0.0009(0.3453)$ \\
\hline$\beta_{11}: 1$ & $0.1788(4.8738)$ & $0.7779(1.4818)$ & $0.4566(1.0430)$ & $0.2930(1.4110)$ & $0.3190(1.6596)$ & $0.0299(0.3321)$ & $0.0526(0.4502)$ \\
\hline$\beta_{12}: 1$ & $0.0983(5.5528)$ & $0.7600(1.3980)$ & $0.4632(0.9917)$ & $0.2787(1.3798)$ & $0.3233(1.7069)$ & $0.0445(0.4024)$ & $0.0408(0.4929)$ \\
\hline & $0.0175(4.8827)$ & $0.0012(0.2373)$ & $0.0053(0.1499)$ & $0.0052(0.4807)$ & $0.0023(0.703)$ & $0.0115(0.0469)$ & $0.0114(0.0428)$ \\
\hline$\beta_{21}^{20}:-1$ & $0.2626(3.9198)$ & $0.0240(0.1881)$ & $0.0415(0.1316)$ & $0.0266(0.4103)$ & $0.0515(0.7089)$ & $0.0003(0.0413)$ & $0.0002(0.0594)$ \\
\hline$\beta_{22}^{21}:-1$ & $0.3090(3.8525)$ & $0.0373(0.1679)$ & $0.0492(0.1490)$ & $0.0639(0.5072)$ & $0.084(0.7683)$ & $0.0054(0.0403)$ & $0.0046(0.0579)$ \\
\hline$\pi_{1}: 0.25$ & $0.1009(0.0886)$ & $0.1257(0.0344)$ & $0.0994(0.0308)$ & $0.0749(0.0276)$ & $0.0908(0.0343)$ & $0.0037(0.0124)$ & $0.0003(0.0158)$ \\
\hline \multicolumn{8}{|c|}{ Case (VI): $\varepsilon \sim N(0,1)$ with $5 \%$ high leverage outliers } \\
\hline$\beta_{10}: 0$ & $0.0763(5.3771)$ & $0.0128(0.7567)$ & $0.0438(1.0701)$ & $0.1006(4.1444)$ & $0.0019(0.5139)$ & $0.0423(0.8197)$ & $0.0046(0.3005)$ \\
\hline & $1.4844(6.1813)$ & $0.4397(1.3832)$ & $0.0892(1.4288)$ & $1.4920(4.6566)$ & $0.2778(0.7660)$ & $0.5649(1.2906)$ & $0.0066(0.3703)$ \\
\hline$\beta_{12}: 1$ & $1.5091(6.2501)$ & $0.4477(1.3542)$ & $0.1050(1.4845)$ & $1.4746(4.6102)$ & $0.2815(0.7487)$ & $0.5472(1.2253)$ & $0.0150(0.3632)$ \\
\hline$\beta_{20}: 0$ & $0.0024(0.0508)$ & $0.0039(0.1774)$ & $0.0029(0.0805)$ & $0.0048(0.0568)$ & $0.0037(0.1261)$ & $0.0005(0.0425)$ & $0.0013(0.0449)$ \\
\hline$\beta_{21}:-1$ & $0.2027(0.1196)$ & $0.0046(0.1412)$ & $0.0082(0.0781)$ & $0.1244(0.0913)$ & $0.0303(0.1008)$ & $0.0701(0.0583)$ & $0.0003(0.0446)$ \\
\hline$\beta_{22}:-1$ & $0.2099(0.1244)$ & $0.0050(0.1365)$ & $0.0030(0.0812)$ & $0.1338(0.0964)$ & $0.0237(0.1078)$ & $0.0677(0.0586)$ & $0.0004(0.0460)$ \\
\hline$\pi_{1}: 0.25$ & $0.0886(0.0102)$ & $0.0930(0.0247)$ & $0.0461(0.0193)$ & $0.0738(0.0089)$ & $0.0709(0.0204)$ & $0.0364(0.0129)$ & $0.0048(0.0123)$ \\
\hline
\end{tabular}

MSE, mean squared error; MLE, maximum likelihood estimations; TLE, trimmed likelihood estimator. 
Table 3 Bias and MSE (in parentheses) of point estimates for $n=100$.

\begin{tabular}{|c|c|c|c|c|c|c|c|}
\hline True & MLE & TLE & Bisquare & Mixregt & Mixregt-MCD & Mixregs & Mixregs-MCD \\
\hline \multicolumn{8}{|c|}{ Case (I): $\varepsilon \sim N(0,1)$} \\
\hline$\beta_{10}: 0$ & $0.0097(0.1228)$ & $0.0020(0.1002)$ & $0.0006(0.1349)$ & $0.0030(0.1340)$ & $0.0008(0.1554)$ & $0.0064(0.1342)$ & $0.0054(0.1496)$ \\
\hline$\beta_{11}: 1$ & $0.0149(0.1251)$ & $0.0735(0.1139)$ & $0.0240(0.1675)$ & $0.0405(0.1513)$ & $0.0546(0.1995)$ & $0.0019(0.1312)$ & $0.0007(0.1611)$ \\
\hline$\beta_{12}: 1$ & $0.0030(0.1298)$ & $0.0747(0.1147)$ & $0.0328(0.1659)$ & $0.0392(0.1501)$ & $0.0580(0.1954)$ & $0.0044(0.1327)$ & $0.0098(0.1648)$ \\
\hline$\beta_{20}: 0$ & $0.0009(0.0188)$ & $0.0026(0.0168)$ & $0.0000(0.0210)$ & $0.0015(0.0218)$ & $0.0029(0.0255)$ & $0.0020(0.0204)$ & $0.0019(0.0218)$ \\
\hline$\beta_{21}:-1$ & $0.0019(0.0195)$ & $0.0275(0.0173)$ & $0.0025(0.0219)$ & $0.0096(0.0209)$ & $0.0107(0.0261)$ & $0.0039(0.0207)$ & $0.0028(0.0256)$ \\
\hline$\beta_{22}:-1$ & $0.0008(0.0197)$ & $0.0294(0.0165)$ & $0.0061(0.0218)$ & $0.0072(0.0202)$ & $0.0090(0.0255)$ & $0.0069(0.0202)$ & $0.0051(0.0245)$ \\
\hline$\pi_{1}: 0.25$ & $0.0006(0.0061)$ & $0.0523(0.0096)$ & $0.0025(0.0113)$ & $0.0117(0.0049)$ & $0.0157(0.0058)$ & $0.0042(0.0064)$ & $0.0026(0.0072)$ \\
\hline
\end{tabular}

Case (II): $\varepsilon \sim \operatorname{slash}(0,1)$

\begin{tabular}{llllllll}
\hline$\beta_{10}: 0$ & $0.0883(67.0279)$ & $0.0005(0.9510)$ & $0.0018(0.9885)$ & $0.0182(2.1725)$ & $0.0104(2.5396)$ & $0.0008(0.6941)$ & $0.0049(0.6992)$ \\
$\beta_{11}: 1$ & $0.8606(72.2127)$ & $0.5882(1.2027)$ & $0.3194(0.9784)$ & $0.1967(4.4901)$ & $0.1811(4.3832)$ & $0.0462(0.6795)$ & $0.0424(0.7862)$ \\
$\beta_{12}: 1$ & $0.8628(49.1993)$ & $0.5832(1.1459)$ & $0.2903(0.9782)$ & $0.1583(3.2396)$ & $0.2100(3.1637)$ & $0.0339(0.6743)$ & $0.0489(0.7677)$ \\
$\beta_{20}: 0$ & $0.1339(84.6754)$ & $0.0034(0.3623)$ & $0.0034(0.3351)$ & $0.0096(1.5744)$ & $0.0138(1.4106)$ & $0.0043(0.0629)$ & $0.0049(0.0883)$ \\
$\beta_{21}:-1$ & $1.7241(64.2070)$ & $0.0364(0.1853)$ & $0.0829(0.2552)$ & $0.0985(1.1663)$ & $0.1289(1.2812)$ & $0.0052(0.0665)$ & $0.0054(0.1086)$ \\
$\beta_{22}:-1$ & $1.6774(64.7592)$ & $0.0491(0.2135)$ & $0.0832(0.2432)$ & $0.1126(1.2056)$ & $0.1139(1.1414)$ & $0.0057(0.0688)$ & $0.0010(0.1151)$ \\
$\pi_{1}: 0.25$ & $0.1798(0.1945)$ & $0.1198(0.0328)$ & $0.0988(0.0351)$ & $0.0795(0.0355)$ & $0.0924(0.0421)$ & $0.0075(0.0260)$ & $0.0113(0.0288)$ \\
\hline
\end{tabular}

Case (III): $\varepsilon \sim t_{1}$

\begin{tabular}{lccccccc}
\hline$\beta_{10}: 0$ & $10.2255(502197.693)$ & $0.0724(7.9262)$ & $0.0025(1.6847)$ & $0.0193(2.6750)$ & $0.0042(2.7491)$ & $0.0165(0.8272)$ & $0.0064(0.9149)$ \\
$\beta_{11}: 1$ & $53.9287(473663.046)$ & $0.2454(6.7949)$ & $0.4377(1.7240)$ & $0.5182(4.3426)$ & $0.5384(3.8060)$ & $0.0625(0.8331)$ & $0.0764(0.9773)$ \\
$\beta_{12}: 1$ & $57.7344(604387.717)$ & $0.1955(6.1361)$ & $0.4510(1.7334)$ & $0.5412(3.3579)$ & $0.5114(3.6211)$ & $0.0624(0.8387)$ & $0.0637(0.9625)$ \\
$\beta_{20}: 0$ & $13.9702(2522395.636)$ & $0.0047(7.0569)$ & $0.0081(0.7474)$ & $0.0597(3.4518)$ & $0.0420(5.3667)$ & $0.0005(0.0544)$ & $0.0047(0.0560)$ \\
$\beta_{21}:-1$ & $92.9882(2734642.730)$ & $0.3511(5.3462)$ & $0.0773(0.5873)$ & $0.1203(3.2147)$ & $0.1104(2.5059)$ & $0.0136(0.0621)$ & $0.0165(0.0712)$ \\
$\beta_{22}:-1$ & $81.6340(2400307.367)$ & $0.3780(5.6155)$ & $0.0908(0.5098)$ & $0.1038(1.3682)$ & $0.2075(6.1631)$ & $0.0122(0.0628)$ & $0.0148(0.0722)$ \\
$\pi_{1}: 0.25$ & $0.2405(0.277)$ & $0.1350(0.0961)$ & $0.1455(0.0543)$ & $0.1159(0.0377)$ & $0.1235(0.0416)$ & $0.0090(0.0336)$ & $0.0141(0.0363)$ \\
\hline Case (IV): $\varepsilon \sim(0,1)$ & & & & & & \\
\hline$\beta_{10}: 0$ & $0.0073(0.2388)$ & $0.0011(0.3148)$ & $0.0020(0.2660)$ & $0.0020(0.1306)$ & $0.0022(0.1452)$ & $0.0059(0.0958)$ & $0.0048(0.1045)$ \\
$\beta_{11}: 1$ & $0.0219(0.2414)$ & $0.3869(0.6129)$ & $0.1957(0.3853)$ & $0.0382(0.1385)$ & $0.0507(0.1716)$ & $0.0268(0.1197)$ & $0.0328(0.1484)$ \\
$\beta_{12}: 1$ & $0.0268(0.2044)$ & $0.4095(0.6287)$ & $0.1973(0.3881)$ & $0.0406(0.1330)$ & $0.0475(0.1663)$ & $0.0363(0.1166)$ & $0.0429(0.1409)$ \\
$\beta_{20}: 0$ & $0.0027(0.0847)$ & $0.0033(0.0484)$ & $0.0010(0.0401)$ & $0.0006(0.0147)$ & $0.0002(0.0177)$ & $0.0020(0.0127)$ & $0.0022(0.0133)$ \\
$\beta_{21}:-1$ & $0.0219(0.0663)$ & $0.0046(0.0271)$ & $0.0202(0.0264)$ & $0.0066(0.0129)$ & $0.0065(0.0155)$ & $0.0018(0.0125)$ & $0.0017(0.0161)$ \\
$\beta_{22}:-1$ & $0.0212(0.0428)$ & $0.0065(0.0274)$ & $0.0228(0.0256)$ & $0.0091(0.0139)$ & $0.0111(0.0173)$ & $0.0026(0.0115)$ & $0.0029(0.0158)$ \\
$\pi_{1}: 0.25$ & $0.0096(0.0063)$ & $0.0425(0.0111)$ & $0.0461(0.0122)$ & $0.0123(0.0043)$ & $0.0144(0.0048)$ & $0.0078(0.0069)$ & $0.0091(0.0081)$ \\
\hline
\end{tabular}

Case $(\mathrm{V}): \varepsilon \sim 0.95 N(0,1)+0.05 N(0,25)$

\begin{tabular}{llllllll}
\hline$\beta_{10}: 0$ & $0.0363(5.8679)$ & $0.0107(0.4219)$ & $0.0034(0.2510)$ & $0.0023(0.3526)$ & $0.0008(0.3908)$ & $0.0018(0.2013)$ & $0.0027(0.2141)$ \\
$\beta_{11}: 1$ & $0.0887(4.9647)$ & $0.6043(0.9888)$ & $0.1826(0.4323)$ & $0.0754(0.4468)$ & $0.0709(0.5583)$ & $0.0050(0.1991)$ & $0.0102(0.2473)$ \\
$\beta_{12}: 1$ & $0.0461(4.9456)$ & $0.6089(0.9898)$ & $0.1928(0.4286)$ & $0.0797(0.4676)$ & $0.0964(0.5888)$ & $0.0077(0.2032)$ & $0.0096(0.2427)$ \\
$\beta_{20}: 0$ & $0.0404(7.0121)$ & $0.0056(0.1193)$ & $0.0022(0.0542)$ & $0.0041(0.1568)$ & $0.0008(0.2250)$ & $0.0016(0.0243)$ & $0.0023(0.0256)$ \\
$\beta_{21}:-1$ & $0.3090(4.3915)$ & $0.0108(0.0553)$ & $0.0165(0.0387)$ & $0.0125(0.1123)$ & $0.0222(0.1786)$ & $0.0079(0.0240)$ & $0.0078(0.0301)$ \\
$\beta_{22}:-1$ & $0.2940(4.3170)$ & $0.0051(0.0524)$ & $0.0136(0.0407)$ & $0.0091(0.1268)$ & $0.0174(0.3255)$ & $0.0062(0.0236)$ & $0.0062(0.0283)$ \\
$\pi_{1}: 0.25$ & $0.0871(0.0992)$ & $0.0781(0.0196)$ & $0.0460(0.0141)$ & $0.0203(0.0095)$ & $0.0238(0.0109)$ & $0.0049(0.0090)$ & $0.0040(0.0103)$ \\
\hline Case (VI): $\varepsilon \sim N(0,1)$ with 5\% high leverage outliers & & & & & \\
\hline$\beta_{10}: 0$ & $0.1116(3.9889)$ & $0.0143(0.5237)$ & $0.0455(0.7972)$ & $0.1189(2.8301)$ & $0.0090(0.1585)$ & $0.0655(0.6801)$ & $0.0082(0.1237)$ \\
$\beta_{11}: 1$ & $1.4606(4.5974)$ & $0.1432(0.8990)$ & $0.3537(1.0996)$ & $1.4982(3.6290)$ & $0.0629(0.2004)$ & $0.7505(1.3577)$ & $0.0019(0.1302)$ \\
$\beta_{12}: 1$ & $1.5307(4.8079)$ & $0.1652(0.8693)$ & $0.3766(1.1550)$ & $1.4945(3.6208)$ & $0.0549(0.1984)$ & $0.7475(1.3744)$ & $0.0050(0.1253)$ \\
$\beta_{20}: 0$ & $0.0104(0.0262)$ & $0.0130(0.0896)$ & $0.0003(0.0426)$ & $0.0105(0.0612)$ & $0.0025(0.0269)$ & $0.0061(0.0196)$ & $0.0003(0.0192)$ \\
$\beta_{21}:-1$ & $0.2252(0.0901)$ & $0.0131(0.0849)$ & $0.0181(0.0337)$ & $0.1373(0.0726)$ & $0.0071(0.0265)$ & $0.1090(0.0441)$ & $0.0010(0.0199)$ \\
$\beta_{22}:-1$ & $0.2277(0.0932)$ & $0.0204(0.0884)$ & $0.0221(0.0371)$ & $0.1352(0.0745)$ & $0.0111(0.0267)$ & $0.1040(0.0409)$ & $0.0018(0.0207)$ \\
$\pi_{1}: 0.25$ & $0.0890(0.0092)$ & $0.0353(0.0107)$ & $0.0006(0.0087)$ & $0.0762(0.0076)$ & $0.0169(0.0062)$ & $0.0624(0.0171)$ & $0.0006(0.0061)$ \\
\hline
\end{tabular}

MSE, mean squared error; MLE, maximum likelihood estimations; TLE, trimmed likelihood estimator. 
Table 4 Bias and MSE (in parentheses) of point estimates for $n=200$.

\begin{tabular}{|c|c|c|c|c|c|c|c|}
\hline True & MLE & TLE & Bisquare & Mixregt & Mixregt-MCD & Mixregs & Mixregs-MCD \\
\hline \multicolumn{8}{|c|}{ Case (I): $\varepsilon \sim N(0,1)$} \\
\hline$\beta_{10}: 0$ & $0.0003(0.0451)$ & $0.0001(0.0364)$ & $0.0001(0.0504)$ & $0.0031(0.0443)$ & $0.0029(0.0474)$ & $0.0035(0.0483)$ & $0.0033(0.0517)$ \\
\hline$\beta_{11}: 1$ & $0.0039(0.0441)$ & $0.0407(0.0376)$ & $0.0024(0.0547)$ & $0.0051(0.0418)$ & $0.0076(0.0493)$ & $0.0037(0.0461)$ & $0.0065(0.0581)$ \\
\hline$\beta_{12}: 1$ & $0.0084(0.0451)$ & $0.0417(0.0374)$ & $0.0010(0.0559)$ & $0.0065(0.0415)$ & $0.0092(0.0497)$ & $0.0084(0.0470)$ & $0.0090(0.0576)$ \\
\hline$\beta_{20}: 0$ & $0.0015(0.0093)$ & $0.0000(0.0075)$ & $0.0014(0.0100)$ & $0.0005(0.0092)$ & $0.0007(0.0094)$ & $0.0029(0.0086)$ & $0.0030(0.0091)$ \\
\hline$\beta_{21}:-1$ & $0.0006(0.0093)$ & $0.0305(0.0086)$ & $0.0018(0.0101)$ & $0.0044(0.0089)$ & $0.0059(0.0104)$ & $0.0012(0.0091)$ & $0.0027(0.0108)$ \\
\hline$\beta_{22}:-1$ & $0.0015(0.0089)$ & $0.0299(0.0084)$ & $0.0001(0.0099)$ & $0.0021(0.0090)$ & $0.0038(0.0105)$ & $0.0003(0.0094)$ & $0.0002(0.0112)$ \\
\hline$\pi_{1}: 0.25$ & $0.0002(0.0026)$ & $0.0581(0.0071)$ & $0.0000(0.0038)$ & $0.0031(0.0022)$ & $0.0043(0.0024)$ & $0.0020(0.0028)$ & $0.0021(0.0032)$ \\
\hline \multicolumn{8}{|c|}{ Case (II): $\varepsilon \sim \operatorname{slash}(0,1)$} \\
\hline$\beta_{10}: 0$ & $0.2238(129.3761)$ & $0.0066(0.4795)$ & $0.0129(0.4322)$ & $0.0154(0.5663)$ & $0.0154(0.9366)$ & $0.0035(0.2805)$ & $0.0050(0.3098)$ \\
\hline$\beta_{11}: 1$ & $1.6322(103.4913)$ & $0.4177(0.6341)$ & $0.0836(0.4221)$ & $0.0852(0.6943)$ & $0.0707(1.6737)$ & $0.0240(0.3173)$ & $0.0348(0.3931)$ \\
\hline$\beta_{12}: 1$ & $1.7944(107.1121)$ & $0.4148(0.6227)$ & $0.0760(0.4140)$ & $0.0886(0.7964)$ & $0.0803(1.2111)$ & $0.0225(0.3114)$ & $0.0294(0.3955)$ \\
\hline$\beta_{20}: 0$ & $0.0987(147.1717)$ & $0.0004(0.1309)$ & $0.0026(0.0980)$ & $0.0058(0.4486)$ & $0.0083(0.3612)$ & $0.0028(0.0209)$ & $0.0008(0.0341)$ \\
\hline$\beta_{21}:-1$ & $2.4287(134.7825)$ & $0.0230(0.0640)$ & $0.0429(0.0570)$ & $0.0314(0.4694)$ & $0.0411(0.3362)$ & $0.0044(0.0292)$ & $0.0009(0.0499)$ \\
\hline$\beta_{22}:-1$ & $2.4348(119.5413)$ & $0.0221(0.0638)$ & $0.0472(0.0604)$ & $0.0321(0.2403)$ & $0.0400(0.5037)$ & $0.0046(0.0279)$ & $0.0027(0.0494)$ \\
\hline$\pi_{1}: 0.25$ & $0.1839(0.2251)$ & $0.0767(0.0158)$ & $0.0540(0.0144)$ & $0.0320(0.0117)$ & $0.0397(0.0158)$ & $0.0063(0.0140)$ & $0.0121(0.0182)$ \\
\hline
\end{tabular}

Case (III): $\varepsilon \sim t_{1}$

$\beta_{10}: 0 \quad 25.0509(1.909716 \mathrm{e}+06)$

$\beta_{11}: 1 \quad 131.7579(2.203641 \mathrm{e}+06)$

$\beta_{12}: 1 \quad 101.4703(1.798630 \mathrm{e}+06)$

$\beta_{20}: 0 \quad 1584.5368(1.254946 \mathrm{e}+10)$

$\beta_{21}:-1 \quad 1150.8596(5.003399 \mathrm{e}+09)$

$\beta_{22}:-1 \quad 3101.5797(4.430502 \mathrm{e}+10)$

$\pi_{1}: 0.25 \quad 0.2413(2.912000 \mathrm{e}-01)$
$0.0184(4.7935)$

$0.1625(3.3181)$

$0.1445(3.4201)$

$0.0478(3.7613)$

$0.1862(2.2135)$

$0.1874(2.2794)$

$0.0894(0.0751)$
$0.0115(0.7171)$

$0.2768(0.9563)$

$0.2997(0.9783)$

$0.0008(0.3362)$

$0.0526(0.2070)$

$0.0424(0.2205)$

$0.1199(0.0353)$
$0.0164(0.5915)$

$0.3669(1.1273)$

$0.3612(1.2457)$

$0.0060(0.2418)$

$0.0363(0.2245)$

$0.0307(0.2821)$

0.0654(0.0199)
$0.0047(0.6980)$

$0.3668(1.4800)$

$0.3758(1.2865)$

$0.0056(0.2008)$

0.0363(0.4318)

0.0440(0.5097)

0.0706(0.0218)
$0.0102(0.2730)$

$0.0552(0.3230)$

$0.0528(0.3290)$

$0.0024(0.0213)$

$0.0028(0.0241)$

$0.0000(0.0248)$

$0.0120(0.0179)$
$0.0078(0.2782)$

$0.0797(0.4144)$

$0.0604(0.3898)$

$0.0025(0.0230)$

$0.0040(0.0292)$

$0.0016(0.0303)$

$0.0164(0.0211)$

Case (IV): $\varepsilon \sim l(0,1)$

\begin{tabular}{|c|c|c|c|c|c|c|c|}
\hline$\beta_{10}: 0$ & $0.0024(0.0480)$ & $0.0028(0.1411)$ & $0.0029(0.0749)$ & $0.0008(0.0313)$ & $0.0005(0.0339)$ & $0.0000(0.0223)$ & $0.0006(0.0346)$ \\
\hline$\beta_{11}: 1$ & $0.0055(0.0481)$ & $0.2040(0.2414)$ & $0.0453(0.0997)$ & $0.0060(0.0302)$ & $0.0056(0.0356)$ & $0.0107(0.0249)$ & $0.0137(0.0423)$ \\
\hline$\beta_{12}: 1$ & $0.0023(0.0551)$ & $0.2173(0.2563)$ & $0.0456(0.0974)$ & $0.0073(0.0293)$ & $0.0092(0.0346)$ & $0.0146(0.0250)$ & $0.0186(0.0441)$ \\
\hline$\beta_{20}: 0$ & $0.0017(0.0485)$ & $0.0029(0.0154)$ & $0.0011(0.0078)$ & $0.0007(0.0053)$ & $0.0008(0.0055)$ & $0.0000(0.0060)$ & $0.0001(0.0062)$ \\
\hline$\beta_{21}:-1$ & $0.0115(0.0077)$ & $0.0049(0.0092)$ & $0.0114(0.0070)$ & $0.0019(0.0050)$ & $0.0026(0.0059)$ & $0.0034(0.0058)$ & $0.0045(0.0068)$ \\
\hline$\beta_{22}:-1$ & $0.0148(0.0200)$ & $0.0014(0.0098)$ & $0.0123(0.0072)$ & $0.0032(0.0053)$ & $0.0037(0.0061)$ & $0.0033(0.0061)$ & $0.0041(0.0070)$ \\
\hline$\pi_{1}: 0.25$ & $0.0036(0.0023)$ & $0.0207(0.0046)$ & $0.0265(0.0036)$ & $0.0057(0.0020)$ & $0.0063(0.0021)$ & $0.0054(0.0028)$ & $0.0066(0.0032)$ \\
\hline \multicolumn{8}{|c|}{ Case $(V): \varepsilon \sim 0.95 N(0,1)+0.05 N(0,25)$} \\
\hline$\beta_{10}: 0$ & $0.0627(5.4191)$ & $0.0003(0.2256)$ & $0.0010(0.0908)$ & $0.0002(0.0786)$ & $0.0005(0.0754)$ & $0.0042(0.0726)$ & $0.0005(0.0767)$ \\
\hline$\beta_{11}: 1$ & $0.0103(4.9720)$ & $0.4269(0.5704)$ & $0.0495(0.1354)$ & $0.0100(0.0845)$ & $0.0129(0.0931)$ & $0.0006(0.0674)$ & $0.0060(0.0811)$ \\
\hline$\beta_{12}: 1$ & $0.0643(4.7922)$ & $0.4246(0.5647)$ & $0.0499(0.1379)$ & $0.0075(0.0821)$ & $0.0086(0.0900)$ & $0.0098(0.0679)$ & $0.0128(0.0859)$ \\
\hline$\beta_{20}: 0$ & $0.0544(6.3131)$ & $0.0029(0.0504)$ & $0.0015(0.0136)$ & $0.0014(0.0117)$ & $0.0010(0.0129)$ & $0.0001(0.0102)$ & $0.0000(0.0108)$ \\
\hline$\beta_{21}:-1$ & $0.2140(3.8070)$ & $0.0014(0.0195)$ & $0.0086(0.0135)$ & $0.0008(0.0112)$ & $0.0009(0.0144)$ & $0.0017(0.0108)$ & $0.0018(0.0129)$ \\
\hline$\beta_{22}:-1$ & $0.2437(4.0257)$ & $0.0015(0.0192)$ & $0.0080(0.0137)$ & $0.0019(0.0111)$ & $0.0019(0.0147)$ & $0.0011(0.0103)$ & $0.0030(0.0125)$ \\
\hline$\pi_{1}: 0.25$ & $0.0574(0.0849)$ & $0.0365(0.0084)$ & $0.0229(0.0063)$ & $0.0013(0.0027)$ & $0.0022(0.0031)$ & $0.0014(0.0038)$ & $0.0007(0.0044)$ \\
\hline \multicolumn{8}{|c|}{ Case (VI): $\varepsilon \sim N(0,1)$ with $5 \%$ high leverage outliers } \\
\hline$\beta_{10}: 0$ & $0.1759(2.6488)$ & $0.0012(0.2241)$ & $0.0576(0.4823)$ & $0.1315(1.8995)$ & $0.0015(0.0496)$ & $0.0717(0.4401)$ & $0.0015(0.0442)$ \\
\hline$\beta_{11}: 1$ & $1.4949(3.5135)$ & $0.0875(0.3978)$ & $0.3566(0.7782)$ & $1.5023(3.0228)$ & $0.0089(0.0493)$ & $0.6859(1.1268)$ & $0.0033(0.0466)$ \\
\hline$\beta_{12}: 1$ & $1.4925(3.5030)$ & $0.0779(0.4179)$ & $0.3686(0.8232)$ & $1.4895(2.9826)$ & $0.0040(0.0522)$ & $0.6703(1.0896)$ & $0.0048(0.0478)$ \\
\hline$\beta_{20}: 0$ & $0.0080(0.0145)$ & $0.0037(0.0366)$ & $0.0006(0.0105)$ & $0.0072(0.0271)$ & $0.0011(0.0096)$ & $0.0013(0.0098)$ & $0.0015(0.0099)$ \\
\hline$\beta_{21}:-1$ & $0.2441(0.0798)$ & $0.0172(0.0465)$ & $0.0259(0.0129)$ & $0.1376(0.0679)$ & $0.0048(0.0104)$ & $0.1002(0.0312)$ & $0.0002(0.0091)$ \\
\hline$\beta_{22}:-1$ & $0.2445(0.0800)$ & $0.0188(0.0460)$ & $0.0266(0.0126)$ & $0.1421(0.0652)$ & $0.0004(0.0104)$ & $0.1035(0.0314)$ & $0.0031(0.0092)$ \\
\hline$\pi_{1}: 0.25$ & $0.0954(0.0098)$ & $0.0070(0.0041)$ & $0.0138(0.0044)$ & $0.0836(0.0080)$ & $0.0042(0.0025)$ & $0.0561(0.0159)$ & $0.0008(0.0026)$ \\
\hline
\end{tabular}

MSE, mean squared error; MLE, maximum likelihood estimations; TLE, trimmed likelihood estimator. 
Table 5 Bias and MSE (in parentheses) of point estimates for $n=400$.

\begin{tabular}{|c|c|c|c|c|c|c|c|}
\hline True & MLE & TLE & Bisquare & Mixregt & Mixregt-MCD & Mixregs & Mixregs-MCD \\
\hline \multicolumn{8}{|c|}{ Case (I): $\varepsilon \sim N(0,1)$} \\
\hline$\beta_{10}: 0$ & $0.0001(0.0123)$ & $0.0001(0.0115)$ & $0.0000(0.0019)$ & $0.0030(0.0098)$ & $0.0028(0.0113)$ & $0.0010(0.0203)$ & $0.0011(0.0213)$ \\
\hline$\beta_{11}: 1$ & $0.0012(0.0131)$ & $0.0290(0.0191)$ & $0.0013(0.0023)$ & $0.0017(0.0083)$ & $0.0037(0.0094)$ & $0.0031(0.0185)$ & $0.0058(0.0225)$ \\
\hline$\beta_{12}: 1$ & $0.0086(0.0124)$ & $0.0312(0.0189)$ & $0.0008(0.0028)$ & $0.0034(0.0070)$ & $0.0039(0.0199)$ & $0.0034(0.0191)$ & $0.0049(0.0226)$ \\
\hline$\beta_{20}: 0$ & $0.0019(0.0012)$ & $0.0000(0.0011)$ & $0.0012(0.0011)$ & $0.0002(0.0013)$ & $0.0000(0.0021)$ & $0.0015(0.0043)$ & $0.0032(0.0044)$ \\
\hline$\beta_{21}:-1$ & $0.0002(0.0011)$ & $0.0313(0.0009)$ & $0.0009(0.0013)$ & $0.0011(0.0010)$ & $0.0023(0.0056)$ & $0.0003(0.0043)$ & $0.0008(0.0051)$ \\
\hline$\beta_{22}:-1$ & $0.0016(0.0065)$ & $0.0181(0.0011)$ & $0.0001(0.0008)$ & $0.0013(0.0011)$ & $0.0016(0.0059)$ & $0.0005(0.0044)$ & $0.0003(0.0051)$ \\
\hline$\pi_{1}: 0.25$ & $0.0001(0.0011)$ & $0.0652(0.0020)$ & $0.0000(0.0011)$ & $0.0024(0.0009)$ & $0.0023(0.0012)$ & $0.0011(0.0012)$ & $0.0017(0.0013)$ \\
\hline \multicolumn{8}{|c|}{ Case (II): $\varepsilon \sim \operatorname{slash}(0,1)$} \\
\hline$\beta_{10}: 0$ & $0.2343(254.24)$ & $0.0070(0.1151)$ & $0.0093(0.2230)$ & $0.0152(0.0654)$ & $0.0155(0.0366)$ & $0.0071(0.0967)$ & $0.0101(0.1025)$ \\
\hline$\beta_{11}: 1$ & $1.7454(223.56)$ & $0.4048(0.0958)$ & $0.0795(0.2149)$ & $0.0757(0.0492)$ & $0.0691(0.9860)$ & $0.0140(0.1103)$ & $0.0177(0.1463)$ \\
\hline$\beta_{12}: 1$ & $1.8329(213.27)$ & $0.3959(0.2146)$ & $0.0631(0.2017)$ & $0.0832(0.0197)$ & $0.0731(0.8741)$ & $0.0050(0.1088)$ & $0.0130(0.1411)$ \\
\hline$\beta_{20}: 0$ & $0.0759(252.34)$ & $0.0003(0.0957)$ & $0.0021(0.0414)$ & $0.0056(0.0178)$ & $0.0083(0.0856)$ & $0.0027(0.0137)$ & $0.0006(0.0153)$ \\
\hline$\beta_{21}:-1$ & $2.6730(257.54)$ & $0.0211(0.0216)$ & $0.0413(0.0372)$ & $0.0311(0.0182)$ & $0.0408(0.0811)$ & $0.0052(0.0155)$ & $0.0008(0.0197)$ \\
\hline$\beta_{22}:-1$ & $2.6970(223.3501)$ & $0.0103(0.0194)$ & $0.0466(0.0419)$ & $0.0309(0.0949)$ & $0.0392(0.1131)$ & $0.0043(0.0155)$ & $0.0061(0.0195)$ \\
\hline$\pi_{1}: 0.25$ & $0.1859(0.2324)$ & $0.0524(0.0028)$ & $0.0532(0.0097)$ & $0.0319(0.0070)$ & $0.0396(0.0084)$ & $0.0063(0.0057)$ & $0.0092(0.0073)$ \\
\hline \multicolumn{8}{|c|}{ Case (III): $\varepsilon \sim t_{1}$} \\
\hline$\beta_{10}: 0$ & $50.3757(6.4951 \mathrm{e}+8)$ & $0.0102(2.5314)$ & $0.0121(0.1130)$ & $0.0101(0.0814)$ & $0.0049(0.1160)$ & $0.0084(0.0835)$ & $0.0081(0.0904)$ \\
\hline$\beta_{11}: 1$ & $265.7957(9.9166 e+8)$ & $0.0941(1.9187)$ & $0.1923(0.9429)$ & $0.2108(0.0830)$ & $0.3234(1.1781)$ & $0.0490(0.1022)$ & $0.8163(0.1304)$ \\
\hline$\beta_{12}: 1$ & $215.9238(10.6280 \mathrm{e}+8)$ & $0.1153(2.0520)$ & $0.2544(0.9559)$ & $0.2134(0.0849)$ & $0.3563(1.2229)$ & $0.0483(0.1070)$ & $0.0601(0.1299)$ \\
\hline$\beta_{20}: 0$ & $2689.9093(6.4192 \mathrm{e}+12)$ & $0.0253(2.0514)$ & $0.0007(0.0129)$ & $0.0031(0.0092)$ & $0.0021(0.1932)$ & $0.0019(0.0097)$ & $0.0024(0.0102)$ \\
\hline$\beta_{21}:-1$ & $2348.1087(9.86884) e+11$ & $0.1153(1.5243)$ & $0.0341(0.0148)$ & $0.0268(0.0100)$ & $0.0292(0.3334)$ & $0.0021(0.0103)$ & $0.0031(0.0120)$ \\
\hline$\beta_{22}:-1$ & $10589.3970(10.6781 \mathrm{e}+12)$ & $0.1184(1.6543)$ & $0.0352(0.0149)$ & $0.0281(0.0098)$ & $0.0513(0.4334)$ & $0.0000(0.0102)$ & $0.0015(0.0121)$ \\
\hline$\pi_{1}: 0.25$ & $0.2414(0.1872)$ & $0.0223(0.0141)$ & $0.1179(0.0374)$ & $0.0633(0.0065)$ & $0.0585(0.0173)$ & $0.0118(0.0075)$ & $0.0168(0.0091)$ \\
\hline \multicolumn{8}{|c|}{ Case (IV): $\varepsilon \sim l(0,1)$} \\
\hline$\beta_{10}: 0$ & $0.0013(0.0914)$ & $0.0031(0.1121)$ & $0.0037(0.0543)$ & $0.0036(0.0488)$ & $0.0023(0.0887)$ & $0.0000(0.0154)$ & $0.0005(0.0254)$ \\
\hline$\beta_{11}: 1$ & $0.0043(0.0962)$ & $0.2037(0.2321)$ & $0.0447(0.0817)$ & $0.0394(0.0674)$ & $0.0495(0.1073)$ & $0.0101(0.0148)$ & $0.0136(0.0325)$ \\
\hline$\beta_{12}: 1$ & $0.0019(0.0026)$ & $0.2169(0.2457)$ & $0.0449(0.0821)$ & $0.0405(0.0513)$ & $0.0461(0.0928)$ & $0.0137(0.0126)$ & $0.0184(0.0329)$ \\
\hline$\beta_{20}: 0$ & $0.0018(0.0087)$ & $0.0017(0.0130)$ & $0.0013(0.0011)$ & $0.0006(0.0138)$ & $0.0002(0.0081)$ & $0.0000(0.0026)$ & $0.0001(0.0036)$ \\
\hline$\beta_{21}:-1$ & $0.0093(0.0046)$ & $0.0035(0.0017)$ & $0.0109(0.0010)$ & $0.0065(0.0096)$ & $0.0061(0.0073)$ & $0.0033(0.0023)$ & $0.0043(0.0031)$ \\
\hline$\beta_{22}:-1$ & $0.0132(0.0089)$ & $0.0009(0.0028)$ & $0.0119(0.0051)$ & $0.0087(0.0113)$ & $0.0101(0.0078)$ & $0.0031(0.0024)$ & $0.0038(0.0047)$ \\
\hline$\pi_{1}: 0.25$ & $0.0033(0.0021)$ & $0.0173(0.0031)$ & $0.0221(0.0025)$ & $0.0117(0.0021)$ & $0.0139(0.0019)$ & $0.0054(0.0011)$ & $0.0052(0.0019)$ \\
\hline
\end{tabular}

Case $(\mathrm{V}): \varepsilon \sim 0.95 N(0,1)+0.05 N(0,25)$

\begin{tabular}{llllllll}
\hline$\beta_{10}: 0$ & $0.1124(0.0204)$ & $0.0003(0.1112)$ & $0.0003(0.0147)$ & $0.0002(0.0415)$ & $0.0005(0.0345)$ & $0.0041(0.0134)$ & $0.0005(0.0141)$ \\
$\beta_{11}: 1$ & $0.1383(0.0405)$ & $0.4023(0.3214)$ & $0.0246(0.0751)$ & $0.0095(0.0481)$ & $0.0123(0.0489)$ & $0.0006(0.0135)$ & $0.0059(0.0164)$ \\
$\beta_{12}: 1$ & $0.1453(0.0444)$ & $0.4018(0.3123)$ & $0.0251(0.0813)$ & $0.0071(0.0425)$ & $0.0074(0.0461)$ & $0.0099(0.0347)$ & $0.0126(0.0174)$ \\
$\beta_{20}: 0$ & $0.0486(0.0037)$ & $0.0021(0.0205)$ & $0.0013(0.0070)$ & $0.0011(0.0061)$ & $0.0009(0.0068)$ & $0.0001(0.0028)$ & $0.0000(0.0029)$ \\
$\beta_{21}:-1$ & $0.0523(0.0044)$ & $0.0010(0.0116)$ & $0.0083(0.0086)$ & $0.0008(0.0056)$ & $0.0009(0.0071)$ & $0.0017(0.0029)$ & $0.0017(0.0032)$ \\
$\beta_{22}:-1$ & $0.0505(0.0040)$ & $0.0013(0.0112)$ & $0.0078(0.0088)$ & $0.0017(0.0054)$ & $0.0017(0.0073)$ & $0.0010(0.0027)$ & $0.0030(0.0031)$ \\
$\pi_{1}: 0.25$ & $0.0375(0.0026)$ & $0.0315(0.0036)$ & $0.0213(0.0051)$ & $0.0009(0.0011)$ & $0.0021(0.0016)$ & $0.0013(0.0012)$ & $0.0007(0.0014)$ \\
\hline Case (VI): $\varepsilon \sim N(0,1)$ with 5\% high leverage outliers & & & & & \\
\hline$\beta_{10}: 0$ & $0.1873(4.0351)$ & $0.0009(0.1219)$ & $0.0632(0.1811)$ & $0.1415(1.1217)$ & $0.0011(0.0216)$ & $0.0651(03112)$ & $0.0064(0.0359)$ \\
$\beta_{11}: 1$ & $1.5082(5.8193)$ & $0.0783(0.1734)$ & $0.3422(0.3216)$ & $1.5131(2.9812)$ & $0.0081(0.0211)$ & $0.6503(1.1170)$ & $0.0030(0.0228)$ \\
$\beta_{12}: 1$ & $1.4889(6.6128)$ & $0.0730(0.1964)$ & $0.3541(0.3917)$ & $1.4521(2.5438)$ & $0.0017(0.0378)$ & $0.6532(0.9896)$ & $0.0026(0.0224)$ \\
$\beta_{20}: 0$ & $0.0182(0.0313)$ & $0.0031(0.0113)$ & $0.0006(0.0083)$ & $0.0071(0.0118)$ & $0.0006(0.0038)$ & $0.0013(0.0087)$ & $0.0006(0.0072)$ \\
$\beta_{21}:-1$ & $0.2495(0.0325)$ & $0.0169(0.0247)$ & $0.0231(0.0067)$ & $0.1369(0.0321)$ & $0.0041(0.0061)$ & $0.1001(0.0213)$ & $0.0010(0.0086)$ \\
$\beta_{22}:-1$ & $0.2512(0.0396)$ & $0.0167(0.0239)$ & $0.0252(0.0071)$ & $0.0890(0.0326)$ & $0.0003(0.0057)$ & $0.1020(0.0207)$ & $0.0015(0.0074)$ \\
$\pi_{1}: 0.25$ & $0.0986(0.0035)$ & $0.0065(0.0017)$ & $0.0132(0.0023)$ & $0.0828(0.0035)$ & $0.0041(0.0012)$ & $0.0541(0.0113)$ & $0.0006(0.0018)$ \\
\hline
\end{tabular}

MSE, mean squared error; MLE, maximum likelihood estimations; TLE, trimmed likelihood estimator. 


\section{A REAL DATA SET ANALYSIS}

In this section, we apply the proposed robust procedure to tone perception data set [47]. In the experiment, a pure fundamental tone was played to a trained musician and electronically generated overtones were added, determined by a stretching ratio. A value of 2 for the stretch ratio corresponds to the harmonic pattern usually heard in traditional definite pitched instruments. The musician was asked to tune an adjustable tone to the octave above the fundamental tone, and a measurement called tuned gives the ratio of the adjusted tone to the fundamental. 150 pairs of (Actual tone ratio, Perceived tone ratio) values are obtained for the same musician. The variable Perceived tone ratio is treated as a response variable and Actual tone ratio as a predictor. Figure 1 shows the scater plot (in left plot) and histogram (in rigth) of tone data. The setup of the experiment indicates two mixture components in the model, and the scatter plot of the data collected from the experiment confirms this point. The right plot in Figure 2 shows that the robust regression based on slash distribution is better than robust regression based normal and $t$ distributions. With the left plot in Figure 2, can be understand the reason of using MRMs, because the traditional models (MLE) is very affected by outlier. Many papers have analyzed this dataset using a mixture of linear regressions framework; see $[48,49,21,27]$. Now we revisit this data set with the aim of expanding the inferential results to the slash distributions. Table 5 reports various fitted models.

From Table 6, we observe that the robust regression based on slash distribution is better than robust regression based normal and $t$ distribution, according to the AIC [50] and BIC [51] criterion.
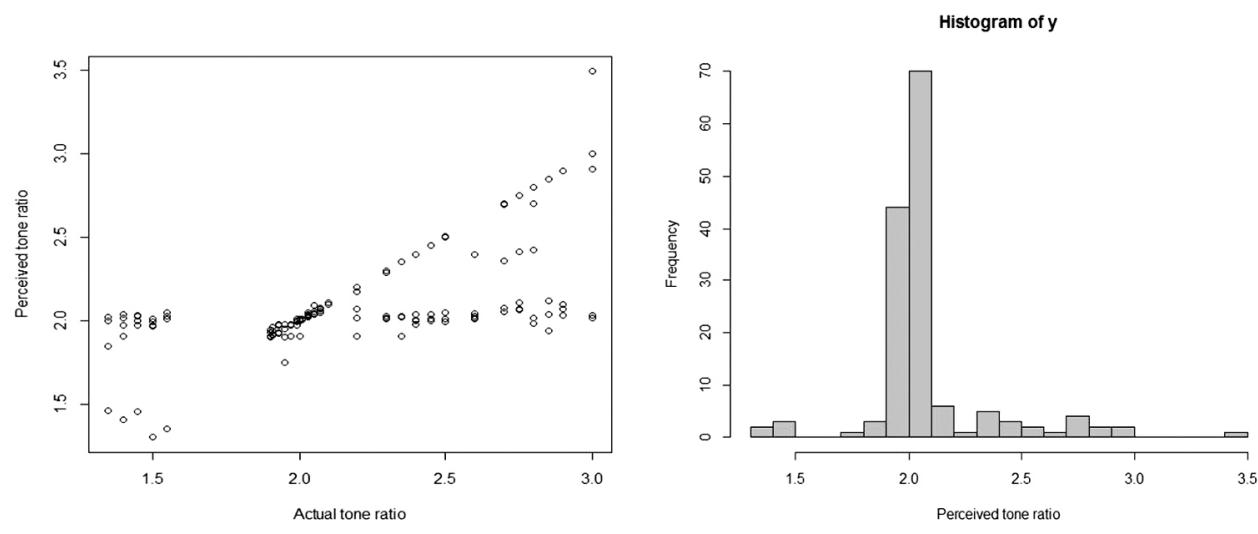

Figure 1 Left plot is the scatterplot of the tone data. Right plot is the histogram of the perceived tone ratio.
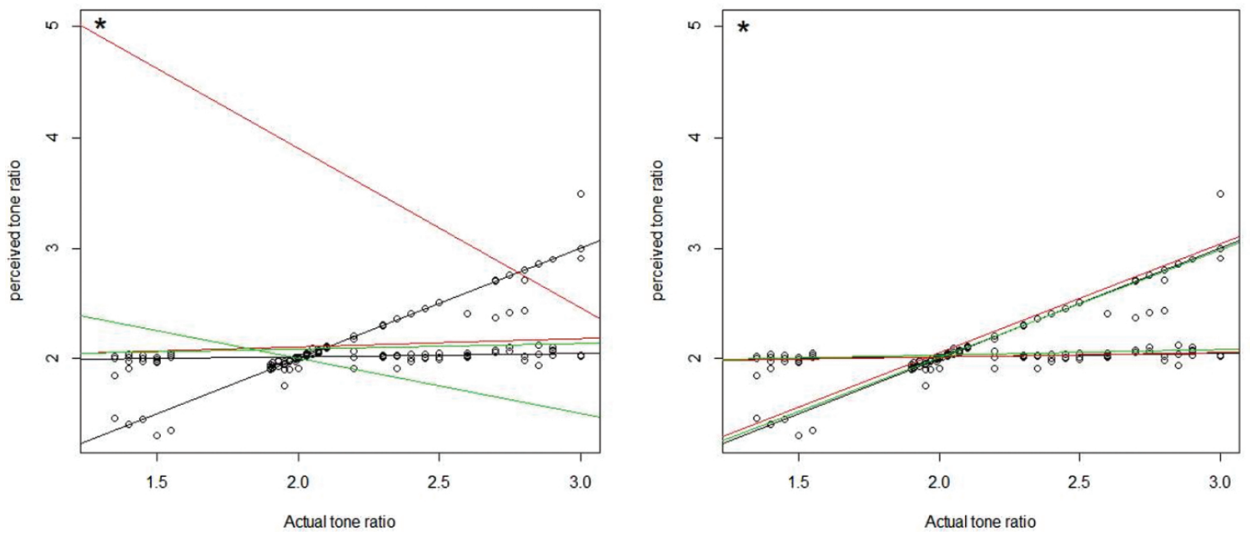

Figure 2 Mixture linear fitting with outlier (1.3,5). In left plot: black line-mixregs, green line-TLE, red line-MLE, in right plot: black line-mixregs, green line-bisquare, red line-mixregt.

Table 6 Fitted various models on the tone perception data set.

\begin{tabular}{lcccccccccccc}
\hline Model & $\hat{\boldsymbol{\beta}}_{\mathbf{0 1}}$ & $\hat{\boldsymbol{\beta}}_{\mathbf{1 1}}$ & $\hat{\boldsymbol{\beta}}_{\mathbf{0 2}}$ & $\hat{\boldsymbol{\beta}}_{\mathbf{1 2}}$ & $\hat{\boldsymbol{\sigma}}_{\mathbf{1}}$ & $\hat{\boldsymbol{\sigma}}_{\mathbf{2}}$ & $\hat{\boldsymbol{q}}_{\mathbf{1}}$ & $\hat{\boldsymbol{q}}_{\mathbf{2}}$ & $\hat{\boldsymbol{\pi}}$ & $\hat{\boldsymbol{\ell}}$ & AIC & BIC \\
\hline Normal & -0.039 & 1.008 & 1.892 & 0.056 & 0.084 & 0.084 & - & - & 0.325 & 107.257 & -200.513 & -179.439 \\
$t$ & 0.006 & 0.998 & 1.978 & 0.017 & 0.011 & 0.011 & 1 & 1 & 0.485 & 202.804 & -387.608 & -360.513 \\
Slash & $\mathbf{0 . 0 0 3}$ & $\mathbf{0 . 9 9 9}$ & $\mathbf{1 . 9 5 4}$ & $\mathbf{0 . 0 2 9}$ & $\mathbf{0 . 0 0 2}$ & $\mathbf{0 . 0 2 0}$ & $\mathbf{0 . 5 6 9}$ & $\mathbf{1 . 4 5 5}$ & $\mathbf{0 . 4 4 3}$ & $\mathbf{2 2 9 . 4 3 6}$ & $\mathbf{- 4 4 0 . 8 7 1}$ & $\mathbf{- 4 1 3 . 7 7 6}$ \\
\hline
\end{tabular}




\section{CONCLUSION}

In this paper, we propose a robust MRM based on the slash distribution by extending the mixture of slash distributions to the regression setting. We suggest some guidelines to further improve the robustness of the proposed model against possible high leverage outliers by adaptively trimming high leverage points. The proposed method was compared to the traditional MLE and some other robust methods by using a simulation study and by using a real data set, it was shown that the results of the robust regression based on slash distribution is better than robust regression based normal and $t$ distributions. Figure 2 also confirms this supremacy.

\section{AUTHORS' CONTRIBUTIONS}

The authors thank the Associate Editor and anonymous reviewers for their useful comments and suggestions on an earlier version of this manuscript which led to this improved one.

\section{Funding Statement}

This work has been conducted by University of Zabol.

\section{REFERENCES}

1. C. da Silva Ferreira, H. Bolfarine, V. H. Lachos, Stat. Methodol. 8 (2011), 154-171.

2. C.R. Cabral, V.H. Lachos, M. Prates, Comput. Stat. Data Anal. 56 (2012), 226-246.

3. C.R. Cabral, V.H. Lachos, C.B. Zeller, J. Multivar. Anal. 124 (2014), 179-198.

4. T.I. Lin, H.J. Ho, C.R. Lee, Stat. Comput. 24 (2014), 531-546.

5. M.O. Prates, C.R. Cabral, V.H. Lachos, J. Stat. Softw. 54 (2013), 12.

6. S.M. Goldfeld, R.E. Quandt, J. Econom. 1 (1973), 3-15.

7. R.E. Quandt, J.B. Ramsey, J. Am. Stat. Assoc. 73 (1978), 730-738.

8. W.S. DeSarbo, W.L. Cron, J. Classif. 5 (1988), 248-282.

9. W.S. DeSarbo, M. Wedel, M. Vriens, V. Ramaswamy, Mark. Lett. 3 (1992), 273-288.

10. S.R. Cosslett, L.-F. Lee, J. Econom. 27 (1985), 79-97.

11. J.D. Hamilton, Econom. 57 (1989), 357-384.

12. T.R. Turner, J. R. Stat. Soc. Ser. C. Appl. Stat. 49 (2000), 371-384.

13. R.B. Arellano-Valle, L.M. Castro, M.G. Genton, H.W. Gómez, et al., Bayesian Anal. 3 (2008), 513-539.

14. M. Liu, G.R. Hancock, J.R. Harring, J. Mod. Appl. Stat. Methods. 10 (2011), 22.

15. A.P. Dempster, N.M. Laird, D.B. Rubim, J. R. Stat. Soc. Ser. B. 39 (1977), 1-38.

16. M. Markatou, Biometrics. 56 (2000), 483-486.

17. H. Shen, J. Yang, S. Wang, in: C. Bussler, D. Fensel (Eds.), Artificial Intelligence: Methodology, Systems, and Applications, Lecture Notes in Computer Science, vol. 3192, Springer, Berlin, Heidelberg, 2004, pp. 208-215.

18. N. Neykov, P. Filzmoser, R. Dimova, P. Neytchev, Comput. Stat. Data Anal. 52 (2007), 299-308.

19. X. Bai, W. Yao, J.E. Boyer, Comput. Stat. Data Anal. 56 (2012), 2347-2359.

20. S. Bashir, E. Carter, Commun. Stat. Theor. Methods. 41 (2012), 3371-3388.

21. W. Yao, Y. Wei, C. Yu, Comput. Stat. Data Anal. 71 (2014), 116-127.

22. A. Azzalini, Scand. J. Stat. 12 (1985), 171-178. https://www.jstor.org/stable/4615982

23. M. Liu, T.I. Lin, Educ. Psychol. Measurement. 74 (2014), 139-162.

24. F.Z. Dogru, O. Arslan, Commun. Stat. Theor. Methods. 46 (2017), 10879-10896.

25. T.I. Lin, J.C. Lee, W.J. Hsieh, Stat. Comput. 17 (2007), 81-92.

26. W. Song, W. Yao, Y. Xing, Comput. Stat. Data Anal. 71 (2014), 128-137.

27. C.B. Zeller, C.R. Cabral, V.H. Lachos, Test. 25 (2016), 375-396.

28. F.Z. Dogru, O. Arslan, Robust Mixture Regression Using Mixture of Different Distributions, Springer, New Delhi, India, 2016, pp. 57-79.

29. F.Z. Dogru, O. Arslan, Rev. Colomb. Estad. 40 (2017), 45-64.

30. F.Z. Dogru, O. Arslan, Commun. Stat. Simul. Comput. 47 (2018), 2184-2196.

31. W.H. Rogers, W. John, J.W. Tukey, Stat. Neerl. 26 (1972), 211-226.

32. R.A. Maronna, R.D. Martin, V.J. Yohai, Robust Statistics: Theory and Methods, John Wiley \& Sons, New York, NY, USA, 2006.

33. K. Kafadar, J. Am. Stat. Assoc. 77 (1982), 416-424.

34. S. Morgenthaler, J.W. Tukey, Configural Polysampling: A Route to Practical Robustness, Wiley, NewYork, NY, USA, 1991. https://www.amazon.com/Configural-Polysampling-Robustness-Probability-Statistics/dp/0471523720

35. P. Bindu, Statistica. 72 (2012), 235-249. https://rivista-statistica.unibo.it/article/view/3645/2996

36. I.C. Alcantara, F.J. Cysneiros, Comput. Stat. Data Anal. 64 (2013), 153-164. 
37. W. Yao, J. Stat. Plan. Inference. 140 (2010), 2089-2098.

38. R.J. Hathaway, Ann. Stat. 13 (1985), 795-800.

39. J. Chen, X. Tan, R. Zhang, Stat. Sin. 18 (2008), 443-465. https://www.jstor.org/stable/24308490

40. G.J. McLachlan, D. Peel, Finite Mixture Models, John Wiley \& Sons, New York, NY, USA, 2000.

41. G.J. McLachlan, T. Krishnan, The EM Algorithm and Extensions, John Wiley \& Sons, New Jersey, USA, 2008.

42. D. MacKay, An Example Inference Task: Clustering, Information Theory, Inference and Learning Algorithms, chap. 20, Cambridge University Press, 2003, pp. 284-292. https://www.inference.org.uk/itprnn/book.pdf

43. T. Louis, J. R. Stat. Soc. Ser. B. 44 (1982), 226-233.

44. K.E. Basford, D.R. Greenway, G.J. McLachlan, D. Peel, Comput. Stat. 12 (1997), 117. https://www.researchgate.net/profile/G_Mclachlan/ publication/37625647_Standard_errors_of_fitted_component_means_of_normal_mixtures/links/09e4150c54f951e160000000/Standarderrors-of-fitted-component-means-of-normal-mixtures.pdf

45. Y. Wei, Robust MRM Using t-Distribution, Master Report, Department of Statistics, Kansas State University, Manhattan, KS, USA, 2012. https://krex.k-state.edu/dspace/bitstream/handle/2097/14110/YanWei2012.pdf?sequence=1\&isAllowed=y

46. P.J. Rousseeuw, B.C. van Zomeren, J. Am. Stat. Assoc. 85 (1990), 633-639.

47. E. Cohen, Music Percept. 1 (1984), 323-349.

48. R.D. DeVeaux, Comput. Stat. Data Anal. 8 (1989), 227-245.

49. D.R. Hunter, D.S. Young, J. Nonparametr. Stat. 24 (2012), 19-38.

50. H. Akaike, in: B.N. Petrov, B.F. Csaki (Eds.), Second International Symposium on Information Theory, Academiai Kiado, Budapest, Hungary, 1973, pp. 267-281. http://amosdevelopment.com/webhelp/index.html?akaike_1973.htm

51. G. Schwarz, Ann. Stat. 6 (1978), 461-464. 
H. Saboori et al. / Journal of Statistical Theory and Applications 19(2) 118-132

131

APPENDIX A

$$
\begin{gathered}
\frac{\partial^{2} \ell_{c}^{*}}{\partial q_{j}^{2}}=-\frac{\sum_{i=1}^{n} z_{i j}}{q_{j}^{2}}, \quad \frac{\partial^{2} \ell_{c}^{*}}{\partial q_{j} \partial \beta_{j}}=\frac{\partial^{2} \ell_{c}^{*}}{\partial \pi_{j} \partial \beta_{j}}=\mathbf{0}_{p \times 1}, \quad \frac{\partial^{2} \ell_{c}^{*}}{\partial q_{j} \partial \sigma_{j}}=\frac{\partial^{2} \ell_{c}^{*}}{\partial q_{j} \partial \pi_{j}}=\frac{\partial^{2} \ell_{c}^{*}}{\partial \pi_{j} \partial \sigma_{j}}=0, \\
\frac{\partial^{2} \ell_{c}^{*}}{\partial \sigma_{j}^{2}}=\sum_{i=1}^{n} z_{i j}\left\{\frac{-3 u_{i}^{2}\left(y_{i}-\mathbf{x}_{i}^{\top} \beta_{j}\right)^{2}}{\sigma_{j}^{4}}+\frac{1}{\sigma_{j}^{2}}\right\}, \quad \frac{\partial^{2} \ell_{c}^{*}}{\partial \beta_{j}^{2}}=-\frac{1}{\sigma_{j}^{2}} \sum_{i=1}^{n} z_{i j} u_{i}^{2} \mathbf{x}_{i} \mathbf{x}_{i}^{\top}, \\
\frac{\partial^{2} \ell_{c}^{*}}{\partial \pi_{j}^{2}}=-\frac{\sum_{i=1}^{n} z_{i j}}{\pi_{j}^{2}}, \quad \frac{\partial^{2} \ell_{c}^{*}}{\partial \sigma_{j} \partial \beta_{j}}=\frac{-2}{\sigma_{j}^{3}} \sum_{i=1}^{n} z_{i j} u_{i}^{2} \mathbf{x}_{i}\left(y_{i}-\mathbf{x}_{i}^{\top} \beta_{j}\right) .
\end{gathered}
$$

APPENDIX B

$$
\begin{gathered}
c_{11}^{j}=-\frac{\sum_{i=1}^{n} p_{i j} u_{i j} \mathbf{x}_{i} \mathbf{x}_{i}^{\top}}{\sigma_{j}^{2}}, \quad c_{22}^{j}=-\frac{\sum_{i=1}^{n} p_{i j}}{q_{j}^{2}}, \quad c_{33}^{j}=\sum_{i=1}^{n} p_{i j}\left\{\frac{-3 u_{i j}\left(y_{i}-\mathbf{x}_{i}^{\top} \beta_{j}\right)^{2}}{\sigma_{j}^{4}}+\frac{1}{\sigma_{j}^{2}}\right\}, \\
c_{44}^{j}=-\frac{\sum_{i=1}^{n} p_{i j}}{\pi_{j}^{2}}, \quad c_{13}^{j}=\frac{-2}{\sigma_{j}^{3}} \sum_{i=1}^{n} p_{i j} u_{i j} \mathbf{x}_{i}\left(y_{i}-\mathbf{x}_{i}^{\top} \beta_{j}\right), \quad c_{31}^{j}=\left(c_{13}^{j}\right)^{\top}, \quad c_{21}^{j}=c_{41}^{j}=\mathbf{0}_{1 \times p}, \\
c_{12}^{j}=c_{14}^{j}=\mathbf{0}_{p \times 1}, \quad c_{23}^{j}=c_{32}^{j}=c_{24}^{j}=c_{42}^{j}=c_{34}^{j}=c_{43}^{j}=0,
\end{gathered}
$$

with

$$
p_{i j}=\frac{\pi_{j} f\left(y_{i}-\mathbf{x}_{i}^{\top} \beta_{j} ; \sigma_{j}, q_{j}\right)}{\sum_{l=1}^{m} \pi_{f} f\left(y_{i}-\mathbf{x}_{i}^{\top} \beta_{l} ; \sigma_{l}, q_{l}\right)}
$$

and

$$
u_{i j}= \begin{cases}\frac{q_{j}^{2}{ }^{\frac{q_{j}}{2}}\left(\frac{\left(y_{i}-\mathbf{x}_{i}^{\top} \beta_{j}\right)^{2}}{\sigma_{j}^{2}}\right)^{-\frac{q_{j}+3}{2}}}{\sigma_{j} \sqrt{\pi} f\left(y_{i}-\mathbf{x}_{i}^{\top} \beta_{j} ; \sigma_{j}, q_{j}\right)} \Gamma\left(\frac{q_{j}+3}{2}\right) G\left(\frac{\left(y_{i}-\mathbf{x}_{i}^{\top} \beta_{j}\right)^{2}}{2 \sigma_{j}^{2}} ; \frac{q_{j}+3}{2}, 1\right), & \text { if } y_{i}-\mathbf{x}_{i}^{\top} \beta_{j} \neq 0, \\ \frac{q_{j}}{q_{j}+3} \frac{1}{\sigma_{j} \sqrt{2 \pi} f\left(0 ; \sigma_{j}, q_{j}\right)}, & \text { if } y_{i}-\mathbf{x}_{i}^{\top} \beta_{j}=0 .\end{cases}
$$

APPENDIX C

$$
v_{11}^{j}=\frac{\sum_{i=1}^{n} z_{i j}^{2} \mathbf{x}_{i}^{\top} \mathbf{x}_{i}\left(y_{i}-\mathbf{x}_{i}^{\top} \beta_{j}\right)^{2} V_{3, i j}}{\sigma_{j}^{4}}, \quad v_{22}^{j}=\sum_{i=1}^{n} z_{i j}^{2} V_{1, i j}, \quad v_{33}^{j}=\frac{\sum_{i=1}^{n} z_{i j}^{2}\left(y_{i}-\mathbf{x}_{i}^{\top} \beta_{j}\right)^{4} V_{3, i j}}{\sigma_{j}^{6}},
$$




$$
\begin{gathered}
v_{12}^{j}=\frac{\sum_{i=1}^{n} z_{i j}^{2} \mathbf{x}_{i}\left(y_{i}-\mathbf{x}_{i}^{\top} \beta_{j}\right) V_{2, i j}}{\sigma_{j}^{2}}, \quad v_{21}^{j}=\left(v_{12}^{j}\right)^{\top}, \quad v_{23}^{j}=\left(v_{32}^{j}\right)^{\top}=\frac{\sum_{i=1}^{n} z_{i j}^{2}\left(y_{i}-\mathbf{x}_{i}^{\top} \beta_{j}\right)^{2} V_{2, i j}}{\sigma_{j}^{3}}, \\
v_{13}^{j}=\frac{\sum_{i=1}^{n} z_{i j}^{2} \mathbf{x}_{i}\left(y_{i}-\mathbf{x}_{i}^{\top} \beta_{j}\right)^{3} V_{3, i j}}{\sigma_{j}^{5}}, \quad v_{31}^{j}=\left(v_{13}^{j}\right)^{\top}, \quad v_{24}^{j}=v_{42}^{j}=v_{44}^{j}=v_{43}^{j}=v_{34}^{j}=0, \\
v_{14}^{j}=\mathbf{0}_{p \times 1}, \quad v_{41}^{j}=\mathbf{0}_{1 \times p},
\end{gathered}
$$

where

$$
\begin{gathered}
V_{1, i j}=\operatorname{Var}\left(\log \left(U_{i}\right) \mid \mathbf{y}, \mathbf{X}, z_{i j}=1\right), \quad V_{2, i j}=\operatorname{Cov}\left(\log \left(U_{i}\right), U_{i}^{2} \mid \mathbf{y}, \mathbf{X}, z_{i j}=1\right) \\
V_{3, i j}=\operatorname{Var}\left(U_{i}^{2} \mid \mathbf{y}, \mathbf{X}, z_{i j}=1\right),
\end{gathered}
$$

it is useful to note that $V_{1, i j}, V_{2, i j}$ and $V_{3, i j}$ can be drive from (17). 